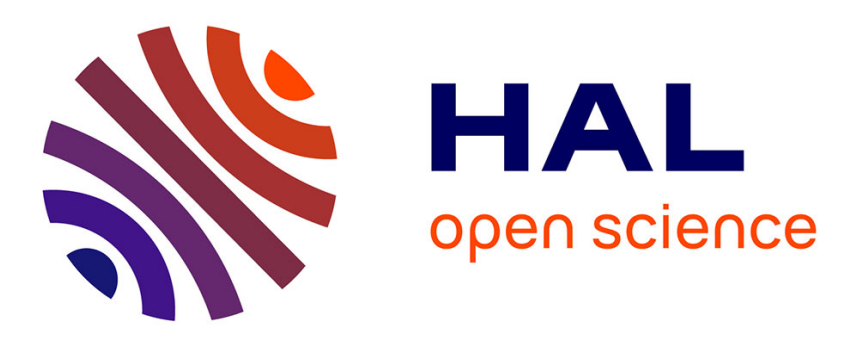

\title{
Two palmitoyl acyltransferases involved sequentially in the biogenesis of the inner membrane complex of Toxoplasma gondii
}

\author{
Sunil Kumar Dogga, Karine Frénal
}

\section{- To cite this version:}

Sunil Kumar Dogga, Karine Frénal. Two palmitoyl acyltransferases involved sequentially in the biogenesis of the inner membrane complex of Toxoplasma gondii. Cellular Microbiology, 2020, 22 (9), 10.1111/cmi.13212 . hal-03040528

\section{HAL Id: hal-03040528 \\ https://hal.science/hal-03040528}

Submitted on 6 Oct 2021

HAL is a multi-disciplinary open access archive for the deposit and dissemination of scientific research documents, whether they are published or not. The documents may come from teaching and research institutions in France or abroad, or from public or private research centers.
L'archive ouverte pluridisciplinaire $\mathbf{H A L}$, est destinée au dépôt et à la diffusion de documents scientifiques de niveau recherche, publiés ou non, émanant des établissements d'enseignement et de recherche français ou étrangers, des laboratoires publics ou privés. 


\title{
Two palmitoyl acyltransferases involved sequentially in the biogenesis of the inner membrane complex of Toxoplasma gondii
}

\author{
Sunil Kumar Dogga | Karine Frénal
}

Department of Microbiology and Molecular Medicine, CMU, Faculty of Medicine,

University of Geneva, Geneva, Switzerland

\section{Correspondence}

Karine Frénal, Laboratoire de Microbiologie Fondamentale et Pathogénicité, UMR5234, University of Bordeaux and CNRS, 146 Rue Léo Saignat, Bat. 3A, Bordeaux Cedex, 33076 , France.

Email: karine.frenal@u-bordeaux.fr

\section{Present address}

Sunil Kumar Dogga, Wellcome Genome

Campus, Wellcome Sanger Institute,

Hinxton, UK

Funding information

Sir Jules Thorn Charitable Overseas Trust reg., Schaan, Grant/Award Number: grant for young researchers 2014; MalarX (ME9782 to DSF)

\begin{abstract}
The phylum Apicomplexa includes a number of significant human pathogens like Toxoplasma gondii and Plasmodium species. These obligate intracellular parasites possess a membranous structure, the inner membrane complex (IMC), composed of flattened vesicles apposed to the plasma membrane. Numerous proteins associated with the IMC are anchored via a lipid post-translational modification termed palmitoylation. This acylation is catalysed by multi-membrane spanning protein S-acyl-transferases (PATs) containing a catalytic Asp-His-His-Cys (DHHC) motif, commonly referred to as DHHCs. Contrasting the redundancy observed in other organisms, several PATs are essential for T. gondii tachyzoite survival; 2 of them, TgDHHC2 and TgDHHC14 being IMC-resident. Disruption of either of these $\mathrm{TgDHHC}$ results in a rapid collapse of the IMC in the developing daughter cells leading to dramatic morphological defects of the parasites while the impact on the other organelles is limited to their localisation but not to their biogenesis. The acyltransferase activity of TgDHHC2 and TgDHHC14 is involved sequentially in the formation of the sub-compartments of the IMC. Investigation of proteins known to be palmitoylated and localised to these sub-compartments identified TgISP1/3 as well as TgIAP1/2 to lose their membrane association revealing them as likely substrates of $\mathrm{TgDHHC} 2$, while these proteins are not impacted by $\mathrm{TgDHHC14}$ depletion.
\end{abstract}

\section{KEYWORDS}

cytoskeleton biogenesis, inner membrane complex, palmitoylation, protein S-acyl transferase,

Toxoplasma gondii

\section{1 | INTRODUCTION}

The phylum Apicomplexa comprises numerous obligate intracellular parasites of medical and veterinary importance. Among them, Toxoplasma gondii establishes a chronic infection in about one third of the human population and can lead to severe complications in immunocompromised individuals or in case of congenital infection. Apicomplexans exhibit an atypical cytoskeleton organised around a membranous structure, named inner membrane complex (IMC), and composed of flattened sacs and a meshwork of intermediate filament- like proteins connected to the cortical microtubules (Mann \& Beckers, 2001). The IMC is central in the biology of these parasites, being critical for their motility, replication, polarity and integrity (Anderson-White et al., 2012). The fast replicating stage of T. gondii, called the tachyzoite, divides by endodyogeny, a process in which the IMC provides the scaffold for the two daughter progenies to develop within the mother cell. In T. gondii and related coccidian parasites, freeze-fracture studies from the 70s revealed a high degree of organisation of the IMC (Dubremetz \& Torpier, 1978; Porchet \& Torpier, 1977). At the apical end, one truncated cone-shaped plate 
constitutes the apical cap in which are anchored, for instance, the acylated proteins TgISP1 (IMC Sub-compartment Protein 1), TgGAP70 (gliding-associated protein $70 \mathrm{kDa}$ ) and TgPhIL1 (photosensitised INA-labelled protein 1) (Beck et al., 2010; Frénal et al., 2010; Gilk et al., 2006). The rest of the IMC is composed of three rows of rectangular plates joined together by sutures and constituting the central and basal sections of the IMC (Figure 1a) (Beck et al., 2010). So far, mainly acylated proteins have been found associated to these regions with TgISP2 and TgISP4 in the central part and TgIAP1 (IMCAssociated Protein 1) in the basal part as well as in the basal ring at the edge of the IMC (Figure 1a) (Beck et al., 2010; Frénal, Marq, Jacot, Polonais, \& Soldati-Favre, 2014; Fung, Beck, Robertson, Gubbels, \& Bradley, 2012). More recently, proximity labelling experiments have been carried out with IMC-resident proteins and identified new proteins localised to the apical cap, central and basal regions, as well as longitudinal or transverse sutures (Chen et al., 2015, 2017; Long, Anthony, Drewry, \& Sibley, 2017; Long, Brown, et al., 2017).
Surprisingly, among them, very few are embedded in the membrane of the organelle by transmembrane domain(s) while several are potentially anchored to the outer leaflet by predicted acylation, and especially by palmitoylation.

Palmitoylation refers to the covalent attachment of a palmitate moiety onto cysteine residues of peripheral and integral membrane proteins via a thioester bond, which increases membrane affinity of the substrates, and thereby modulating their localisation, conformation, stability and interaction. Palmitoylation is the only acylation to be enzymatically reversible and thus allows a dynamic regulation of cellular processes (Blaskovic, Blanc, \& van der Goot, 2013; Linder \& Deschenes, 2007). It is widespread in eukaryotes and represents around $10 \%$ of the human proteome (Blanc et al., 2015). In T. gondii, palmitoylation has been proved to be critical for cell division (Beck et al., 2010), pellicle integrity (Frénal et al., 2010), egress of the parasites from the infected host cell (McCoy et al., 2017) as well as for the apical positioning of secretory organelles called rhoptries whose (a)

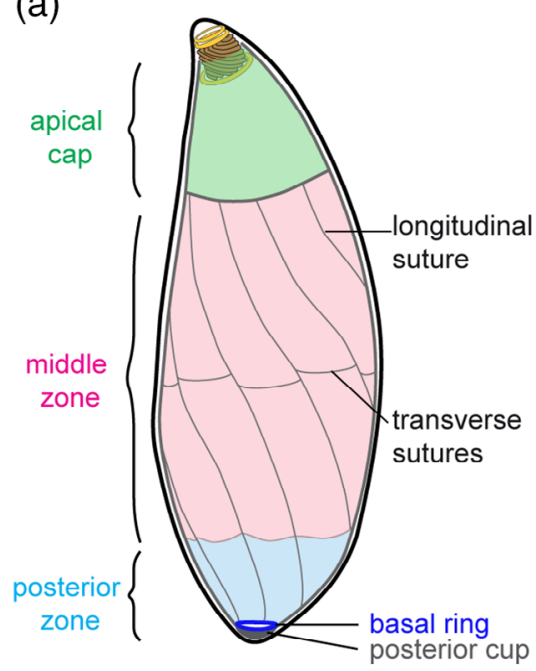

(b)

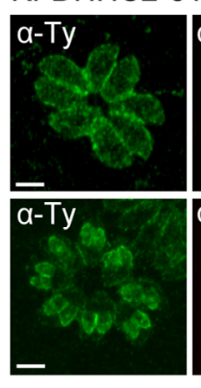

(c) KI-DHHC14-3Ty / KI-ISP3-YFP
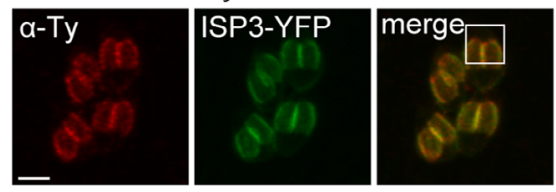

KI-DHHC14-3Ty
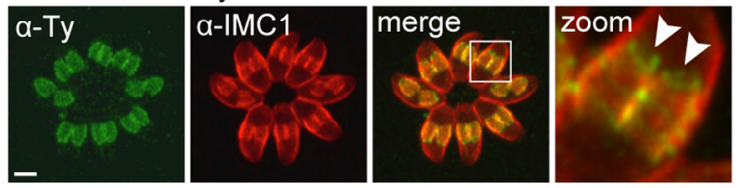

(d) KI-DHHC14-3Ty
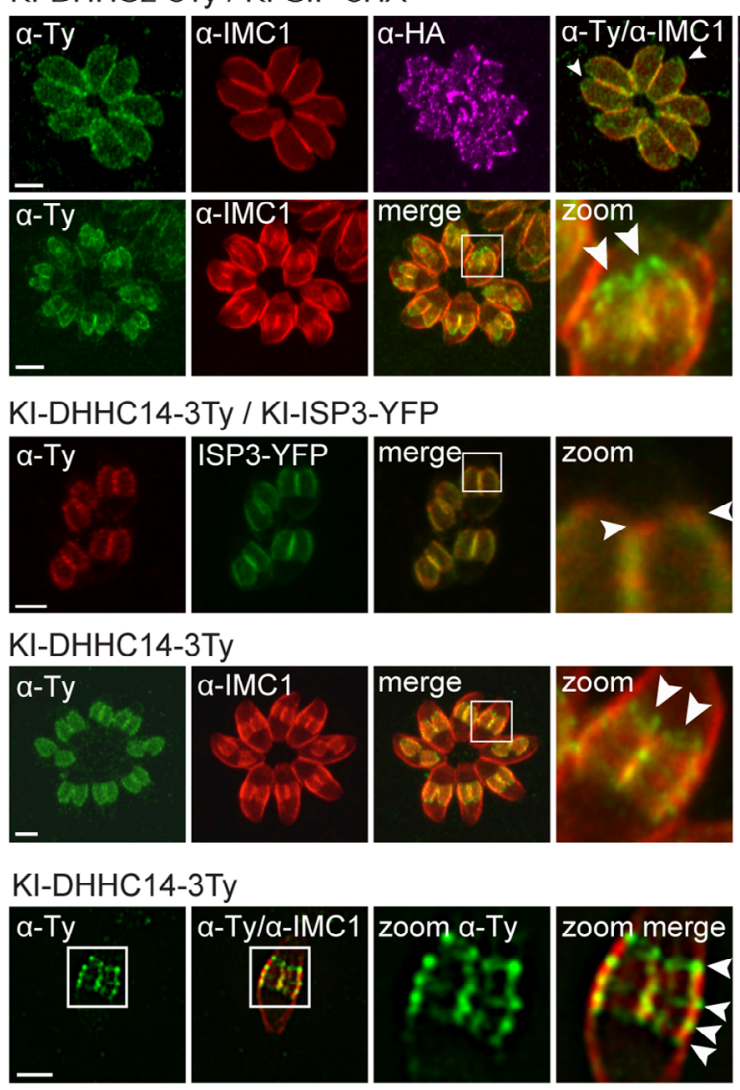
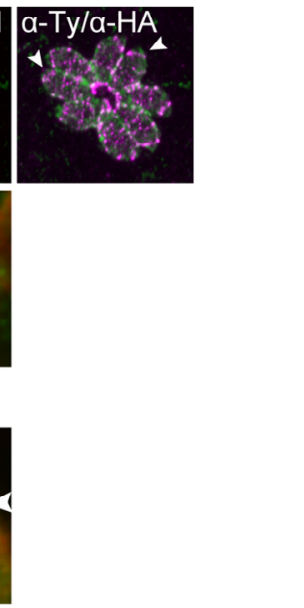
content is secreted at the time of invasion and participates in host cell penetration and establishment of the intracellular lifestyle (Beck et al., 2013; Mueller et al., 2013). Overall, the global analysis of palmitoylated proteins (palmitoylomes) in T. gondii and the related parasite Plasmodium falciparum revealed the presence of over 300 and 400 proteins, respectively, including previously experimentally confirmed ones (Foe et al., 2015; Jones, Collins, Goulding, Choudhary, \& Rayner, 2012). This is significantly more than the palmitoylomes reported before for other unicellular eukaryotes and suggests a large use of this post-translational modification by the Apicomplexans to regulate critical processes (Emmer et al., 2011; Roth et al., 2006). Palmitoylation is mediated by multi-membrane spanning protein $\mathrm{S}$ acyltransferases (PATs) containing a catalytic Asp-His-His-Cys motif facing the cytoplasm and thus commonly referred to as DHHCs (Linder \& Deschenes, 2007). The recently elucidated structure of two DHHCs from human and zebrafish established that the active site, catalysing the thioester exchange from the palmitoyl-coA to the substrate, is located at the membrane-cytosol interface and hence explains why the palmitoylated sites of integral protein substrates reside close to the transmembrane domains (Rana et al., 2018).

T. gondii genome encodes $18 \mathrm{DHHCs}, 16$ of them being expressed in the tachyzoite stage (Frénal et al., 2013). As in other organisms, including humans, most of the DHHCs have been localised to the Golgi apparatus, the endoplasmic reticulum and the plasma membrane but three of them were found in the membrane of apicomplexan-specific organelles, TgDHHC2 and TgDHHC14 in the IMC and TgDHHC7 in the rhoptries (Beck et al., 2013; Frénal et al., 2013). Surprisingly, and in contrast to other organisms in which none of the PATs were individually essential (Emmer et al., 2011; Roth et al., 2006), five DHHCs have been refractory to deletion in $T$. gondii including the three aforementioned ones (Frénal et al., 2013). In this study, we investigate the role of TgDHHC2 and TgDHHC14 for the biology of the tachyzoite. These two PATs are conserved across the phylum Apicomplexa and likely not redundant within the IMC (Frénal et al., 2013; Wetzel et al., 2015). While TgDHHC2 appears as a canonical PAT predicted to have four transmembrane domains with the catalytic region located between the second and the third transmembrane domain, TgDHHC14 exhibits a longer $\mathrm{N}$-terminus encompassing ankyrin repeats. PfDHHC1, the homologue of TgDHHC14 in P. falciparum, has been localised to the IMC of the blood stages, following the PfISP1 and PfISP3 staining during schizogony (Wetzel et al., 2015). In addition, during gametocytogenesis, PfDHHC1 appears in the transversal sutures of the developing IMC. Surprisingly, in P. berghei, $\mathrm{PbDHHC2}$, the homologue of TgDHHC2, is cytoplasmic in the blood stages and ookinete (Santos et al., 2015). PbDHHC2 is likely essential since the gene could not be disrupted but a promoter swap cell line revealed its critical role for the ookinete development, downstream of the fertilisation step, suggesting an involvement in the morphogenesis of this zygote stage. Both PfDHHC2 and PfDHHC1 appear nonmutable in a piggyBac transposon screen of the $P$. falciparum genome (Zhang et al., 2018), suggesting a conserved and essential role of these PATs in apicomplexan parasites.

Here, we show that TgDHHC2 and TgDHHC14 have distinct localisations within the IMC of the tachyzoite. Depletion of either
TgDHHC2 or TgDHHC14 severely compromised IMC biogenesis without impacting the replication process and the biogenesis of other organelles. The collapse of the IMC is due to the loss of the acyltransferase activity of the enzymes. Investigation of proteins of the IMC known to be palmitoylated identified proteins of distinct sub-compartments as probable substrates of $\mathrm{TgDHHC} 2$, while no substrates of TgDHHC14 have been identified.

\section{2 | RESULTS}

\section{1 | Two PATs localised into distinct sub- compartments of the IMC}

To refine the timing of expression and localisation of TgDHHC2 and TgDHHC14 in T. gondii tachyzoite, the strains endogenously tagged with 3Ty by knock-in strategy have been scrutinised by immunofluorescence assay (IFA) using different markers associated with the IMC. TgDHHC2 was detected in mature parasites (Figure $1 \mathrm{~b}$ ) as well as in dividing parasites in which the protein is mainly concentrated in the growing daughter cells. TgDHHC2 localises to the three subcompartments of the IMC as shown in mature parasites by its staining that extends up to the apical end beyond the staining of TgIMC1 that is excluded from the cap and TgSIP (Stripes IMC Protein, [Lentini et al., 2015]) which decorates the transverse sutures of the IMC plates and therefore delineates the base of the apical cap (Figure 1b, upper panel). This localisation is more clearly visible during the budding of the daughter cells in which the staining of TgDHHC2 is detected in the apical cap before the appearance of TgISP3 (Figure S1a in Data S1) and above TgIMC1 (Figure 1b, lower panel). In contrast, TgDHHC14 is nearly undetectable in mature parasites and only visible in the daughter cells where its staining always starts as a ring that elongates along the developing progenies (Figure S1b in Data S1). Costaining with TgISP3 and TgIMC1 (Figure 1c) shows that TgDHHC14 is absent from the apical cap and exhibits a non-homogenous signal in the rest of the IMC. Super-resolution images from structured illumination microscopy (SR-SIM) revealed that TgDHHC14 specifically localises to the transverse sutures of the IMC plates (Figure 1d). In addition, both TgDHHC2 and TgDHHC14 appear before the staining of TgIMC1 is observed in the budding parasites (Figure S1 in Data S1).

These data show that the two palmitoyl transferases of the IMC, TgDHHC2 and TgDHHC14, are mainly expressed during the division process of the parasites and have distinct localisations within the IMC, with TgDHHC2 found in the full organelle while TgDHHC14 is restricted to the transverse junctions of the IMC.

\subsection{IgDHHC2 and TgDHHC14 are critical for the life cycle of $\mathrm{T}$. gondii tachyzoite}

We previously failed to individually disrupt these genes, suggesting that despite some overlapping localisation between TgDHHC2 and TgDHHC14, these enzymes are likely not redundant and have a 
different substrate specificity within the IMC (Frénal et al., 2013). Therefore, to investigate their function, inducible knock-down (iKD) cell lines have been generated by replacing the endogenous promoter with a tetracycline-repressive promoter using a CRISPR-based genome editing strategy to enhance the integration (Figure S2a,b and Table S2 in Data S1). Subsequently, a 3Ty tag has been added to the $3^{\prime}$ end of the coding sequence by knock-in. The localisation of TgDHHC2 and TgDHHC14 is maintained in these strains (Figure S2c in Data S1) and their expression is tightly down-regulated under anhydrotetracycline (ATc) treatment, reaching a drastic reduction after $16 \mathrm{hr}$ of treatment (Figure 2a) and undetectable levels by western blot within $24 \mathrm{hr}$ for TgDHHC14 and $48 \mathrm{hr}$ for TgDHHC2 (Figure 2b). Depletion of each PAT strongly impairs the growth of the parasites as shown by plaque assays performed on TgDHHC2-iKD, TgDHHC14-iKD and the parental $\triangle \mathrm{KU} 80$ strains (Figure 2c). In contrast to the control, very small (TgDHHC14-iKD + ATc) or no plaques (TgDHHC2-iKD + ATc) were visible on the fibroblast monolayer after 9 days in presence of ATc, indicating a defect in one or more steps of the tachyzoite lytic cycle.

Altogether, these results confirm the critical nature of TgDHHC2 and $\mathrm{TgDHHC14}$ for the tachyzoite survival that was previously suggested by the inability to knock-out these genes (Frénal et al., 2013). It also corroborates the low fitness score $(-4.36$ and -3.21 , respectively) attributed to these genes in the recent CRISPRbased genome-wide screen performed in T. gondii tachyzoite (Sidik et al., 2016).

\section{3 | Depletion of TgDHHC2 or TgDHHC14 alters the IMC biogenesis but not the replication process and the biogenesis of the other organelles}

We examined the intracellular development of TgDHHC2- and TgDHHC14-depleted tachyzoites by IFAs after $16 \mathrm{hr}$ of ATc treatment. Since the two enzymes are localised to the IMC, we first focused our attention on the fate of the IMC during the cell division process using the transmembrane protein TgGAP50 endogenously tagged at its C-terminus with a YFP. In both knock-down strains, initiation of new daughter cells can be observed with the budding occurring in random orientations. However, TgGAP50 staining highlights a strong alteration of the biogenesis and morphology of the IMC later on as well as a strong defect in parasite organisation within the parasitophorous vacuole (Figure 3a). In addition, although the nucleus divides, it is not always associated to an IMC structure that usually encapsulates the newly formed progenies (Figure 3a, arrowheads). Nonetheless, the nuclei remain associated with the centrosomes as shown by the centrin 1 staining (TgCEN1, Figure S3a in Data S1). The IMC of the daughter cells likely collapses earlier in TgDHHC2-depleted parasites than in TgDHHC14-depleted since we do not observe very advanced progenies in this cell line compared to the TgDHHC14 knock-down. In both cases, with the successive rounds of division, the shape of the parasites is more and more altered preventing their normal proliferation in the host cell monolayer. Of note, we sometimes observed intact parasites in the case of TgDHHC14 knock-down cell line that could explain the very small plaques monitored previously. This could be either due to a leakiness of the inducible system in which the residual level of enzyme could be sufficient to ensure the PAT activity or the critical but not totally essential function of TgDHHC14.

In parallel, we examined other organelles that are packaged in the developing daughter cells in a timely orchestrated process during the cell division (Nishi, Hu, Murray, \& Roos, 2008). Secretory organelles such as micronemes and rhoptries are made de novo at each round of division. After their trafficking through the endoplasmic reticulum (ER) and the Golgi apparatus, the secreted proteins are sorted to the apical micronemes or rhoptries through the endosome-like compartment (ELC) (Nishi et al., 2008; Sangaré et al., 2016). Staining of TgMIC2 in the micronemes and TgROP7 in the rhoptries shows that the biogenesis of these organelles is not impacted by the depletion of either TgDHHC2 or TgDHHC14 and they remain fairly associated with apical structures within the deformed parasites (Figure S3a,b in Data S1). Organelles that are inherited during the cell division such as the apicoplast and the mitochondrion were also looked at. In both TgDHHC2- and TgDHHC14-depleted parasites, the apicoplast divides and is most of the time found in close proximity to the nucleus (Figure S3c in Data S1), likely because the organelle associates with the centrosomes during division (Striepen et al., 2000). We also checked the fate of the mitochondrion in both knock-down cell lines since this organelle was reported to make contact sites with the IMC (Ovciarikova, Lemgruber, Stilger, Sullivan, \& Sheiner, 2017) and to be inherited late during the division cycle, crawling along the IMC of the daughter cells (Nishi et al., 2008). Surprisingly, no problem of the mitochondrion inheritance was observed $16 \mathrm{hr}$ post ATc treatment (Figure S3d in Data S1).

The IMC is critical to maintain the shape of the parasite since it is connected to the cortical microtubules, likely through transmembrane proteins of the GAPM (glideosome-associated protein with multiplemembrane spans) family (Bullen et al., 2009; Harding et al., 2019). Staining of the microtubules with the anti-acetylated $\alpha$-tubulin revealed that they emerge with the growing daughter cells (Figure 3b), likely associated with the IMC previously observed with GAP50-YFP. However, again, no elongated daughter cells stained for the microtubules are observed for TgDHHC2-depleted parasites in contrast to TgDHHC14 knock-down parasites.

To have a better understanding of the morphology defect of the down-regulated parasites, the two strains were observed by electron microscopy after $24 \mathrm{hr} \pm$ ATc (Figure 3c and Figure S4 in Data S1). In both cases, $\mathrm{TgDHHC}$-depleted parasites are multinucleated cells containing micronemes, rhoptries, dense granules and mitochondria. However, they present a strong alteration of the IMC which is discontinued and detached from the plasma membrane at the level of the mother cell pellicle. Fragments of IMC still connected to microtubules are also observed floating in the cytoplasm (Figure 3c).

Taken together, the close examination of the parasite morphology shows that depletion of TgDHHC2 or TgDHHC14 induces strong defects in the IMC biogenesis with a collapse of the structure in the developing daughter cells likely occurring earlier in the case of 
TgDHHC2 knock-down compare to TgDHHC14. Despite this severe defect, the cell division process as well as the biogenesis of the other organelles remain apparently unaffected.

\subsection{The loss of acyl-transferase activity is responsible for the collapse of the IMC}

To determine whether TgDHHC2 and TgDHHC14 are active enzymes and if the observed phenotypes were due to a loss of the acyltransferase activity, the two iKD strains were complemented with either the wild type TgDHHC (cpt-DHHC2/14-WT) or with a mutant in which the DHHC motif was substituted by DHHS (cptDHHC2-C194S and cpt-DHHC14-C618S), a point mutation preventing the acyl-transferase activity (Lobo, Greentree, Linder, \& Deschenes, 2002; Zhang, Wu, Kelly, Nurse, \& Hang, 2013). An additional complementation was performed for TgDHHC14 with a copy lacking the ankyrin repeats (cpt-DHHC14- $\triangle \mathrm{ANK}$ ). All these complemented constructs were inserted into the UPRT locus, expressed under the control of their endogenous promoter and tagged with 4 c-Myc (Figure S5a and Table S2 in Data S1).

For each PAT, the complemented proteins were all expressed at similar levels except TgDHHC14- $\triangle$ ANK (Figure 4a) that was well integrated in the genome (Figure S5b,c in Data S1) but likely not targeted to the IMC and therefore unstable and never detected either by western-blot or by IFA. In contrast, the WT and the catalytically dead mutants were localised at the IMC like the endogenously tagged copies (Figure 4b). In TgDHHC2- and TgDHHC14-depleted parasites, only the WT but not the mutated version of the enzyme was able to prevent the collapse of the IMC as shown by IFA (Figure 4b). In accordance, plaque assays performed over 7 days with the complemented strains showed that the growth phenotype of TgDHHC2 and TgDHHC14 depletion can be rescued by the WT copy of TgDHHC2 and TgDHHC14 but not with the single point mutants (Figure 4c).

These results indicate that TgDHHC2 and TgDHHC14 are active enzymes of the IMC and that the collapse of the structure observed upon their depletion is therefore likely due to the non-palmitoylation of substrates contributing to the biogenesis of the IMC.

\subsection{Exploration of potential substrates of TgDHHC2 and TgDHHC14}

To investigate potential substrates of $\mathrm{TgDHHC} 2$ and $\mathrm{TgDHHC} 14$, we focused on proteins known to be palmitoylated and localised at the IMC. We first followed the acylated proteins recruited to the different sub-compartments of the IMC by myristoylation and palmitoylation of their $\mathrm{N}$-terminus, namely the early marker for bud formation TgISP1 at the apical cap, and the later markers TgISP2 in the middle zone and TgISP3 in the central and posterior regions of the IMC (Beck et al., 2010). Upon TgDHHC2 depletion, TgISP1 signal almost totally disappears from the parasites as shown by IFA (Figure 5a). Sequential fractionations corroborate this phenomenon with a total amount of
TgISP1 highly reduced suggesting a strong destabilisation of the protein. Concomitantly, TgISP1 becomes partially soluble in PBS and disappears from the pellet fraction likely because in absence of TgDHHC2, TgISP1 is not palmitoylated and therefore its myristoylation is not enough to strongly anchor the protein to the IMC membrane (Figure 5b). Similarly, TgISP2 and TgISP3 signal associated to membranous material decreases upon TgDHHC2 depletion and appears partially cytoplasmic by IFA (Figure $5 \mathrm{a}$ ). However, no variation of the total amount of protein can be observed in the fractionation experiments and only TgISP3 profile changes with ATC treatment with less protein found in the pellet and more in the soluble fraction (Figure 5b). In contrast, upon depletion of TgDHHC14, the anchoring of TgISP1 at the apical cap is still observed as well as TgISP2 and TgISP3 along the parasite except at the basal pole (Figure 5a). The profiles of TgISP1, TgISP2 and TgISP3 do not change during sequential fractionations in the TgDHHC14-iKD strain treated or not with ATc (Figure 5b).

These results suggest that TgISP1 and TgISP3 are substrates of TgDHHC2 since their localisation and solubility are impacted upon TgDHHC2 depletion while none of the TgISPs seem to be substrates of TgDHHC14.

We then examined IMC proteins that are only palmitoylated. The only reported protein is TgIAP1 (IMC-associated protein) that is part of the MyoC-glideosome at the basal pole of the parasite and anchored in the basal sub-compartment of the IMC by $\mathrm{N}$-terminal palmitoylation (Frénal et al., 2014). We also identified a distant homologue of TgIAP1 that we named TgIAP2 (TGGT1_297130) that presents no particular domain or motif but four predicted palmitoylation sites that are conserved with TgIAP1 (Figure S6 in Data S1). In addition, TgIAP2 is found in the T. gondii palmitoylome like TgIAP1 (Foe et al., 2015). TgIAP1 and TgIAP2 have been endogenously tagged in both TgDHHC2- and TgDHHC14-iKD strains. TgIAP1 localises to the basal pole as expected, while TgIAP2 localises to the IMC but is absent from the apical cap (Figure 6a). Strikingly, upon depletion of TgDHHC2, TgIAP1 signal completely disappears and the signal of TgIAP2 associated with membranes decreases, as observed by IFA. We observe a concomitant change of protein profile in sequential fractionations, with both proteins visible in the PBS soluble fraction. In contrast, with depletion of TgDHHC14, the membrane association of TgIAP1 and TgIAP2, as seen by IFA, as well as the protein profile in the fractionations appears unaltered. These results suggest that both TgIAP1 and TgIAP2 could be substrates of TgDHHC2.

\section{3 | DISCUSSION}

The large repertoire of PATs in Apicomplexa likely highlights the importance of the role of palmitoylation in these parasites. This assumption is further supported by the reported palmitoylomes of $T$. gondii and $P$. falciparum that identified more than 300 proteins involved in multiple aspects of the parasite biology (Caballero et al., 2016; Foe et al., 2015; Jones, Collins, et al., 2012; Jones, Tay, \& Rayner, 2012). However, there is comparatively little knowledge 
about the enzyme-substrate relationships. The rhoptry-localised protein named armadillo repeat only (TgARO), anchored to the surface of the organelle by acylation, is the only substrate shown to be modified by the conserved $\mathrm{TgDHHC7}$, also localised in the membrane of the rhoptry (Beck et al., 2013; Frénal et al., 2013; Mueller et al., 2013). Here, we have investigated the functionality of two other conserved PATs, TgDHHC2 and TgDHHC14, and explored some potential substrates.

In tachyzoites, these two enzymes are mainly expressed in the IMC of the budding daughter cells, but with restricted subcompartmentalisation. TgDHHC2 spans the entire IMC like other transmembrane domain proteins of the IMC such as TgGAP40 and TgGAP50. On the other hand, TgDHHC14 is restricted to the transverse sutures of the IMC like its counterpart in $P$. berghei gametocytes (Wetzel et al., 2015). These latest orthologs constitute the only transmembrane proteins identified in these sutures and it remains to be determined how they can be specifically targeted to these structures.
The distinction of their spatial localisation suggests that they plausibly act on different substrates, though some overlapping substrate specificity cannot be ruled out. TgDHHC2 is observed before the signal of TgISP3 was detected while both TgDHHC2 and TgDHHC14 appear before the staining of TgIMC1. These results give a first insight on the timing of recruitment of IMC-associated proteins during the assembly of the organelle.

We uncovered the critical role of TgDHHC2 and TgDHHC14 for the proper biogenesis of the tachyzoite IMC during endodyogeny. A close examination of all the organelles showed that neither of them appears to be required for the division of the nucleus, the apicoplast and the mitochondrion. Their depletion has also no impact on the biogenesis of the micronemes and rhoptries although their localisation is altered since the integrity of the parasites is lost. Depletion of $\mathrm{TgDHHC} 2$ or TgDHHC14 induces a strong defect in the IMC biogenesis that is dependent on the palmitoyl transferase activity. In both cases, initiation of the daughter cell budding can be observed but the (a)

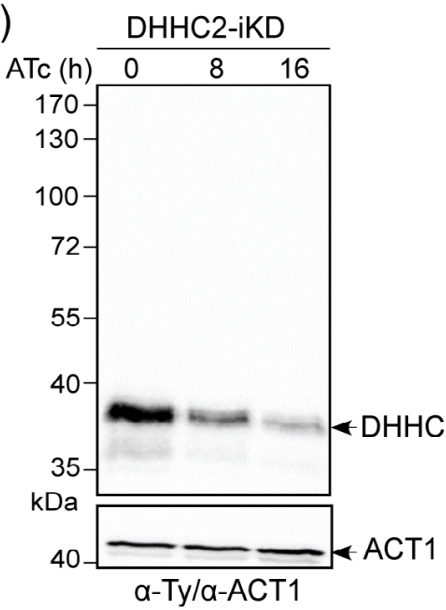

(b)

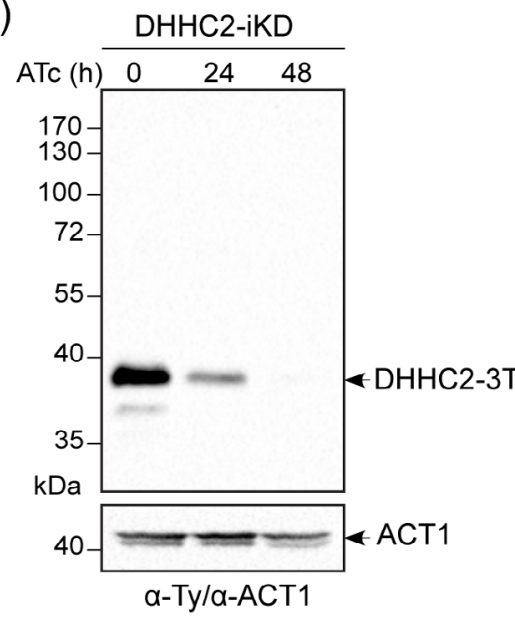

DHHC14-iKD
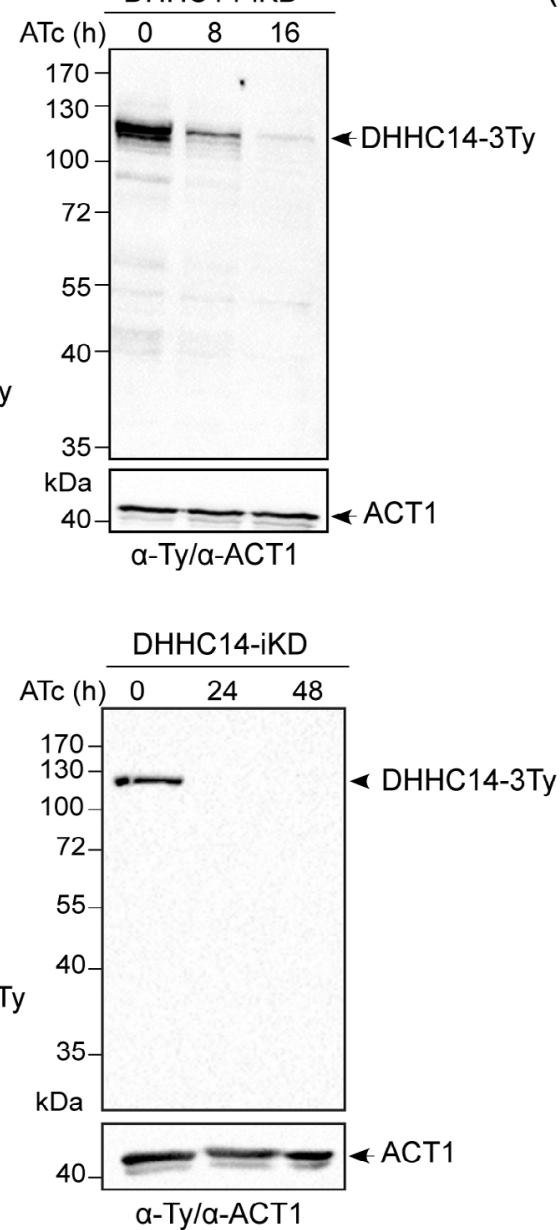

(c)
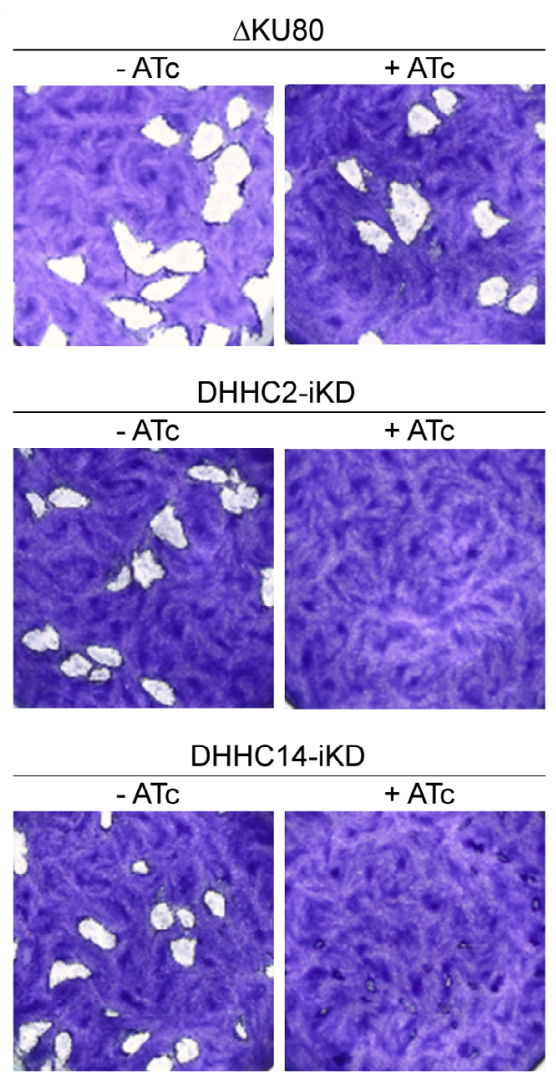

FIGURE 2 DHHC2 and DHHC14 are critical for the lytic cycle of the tachyzoite. (a) The inducible knock-down of TgDHHC2 and TgDHHC14 (TgDHHC2-3Ty-iKD and TgDHHC14-3Ty-iKD), detected at the expected size of 40 and $110 \mathrm{kDa}$, respectively by western-blot and tightly downregulated upon addition of anhydrotetracycline (ATc) with almost no protein detectable after $16 \mathrm{hr}$ of treatment. (b) TgDHHC2 is undetectable at $48 \mathrm{hr}$ of ATc treatment while TgDHHC14 is already not detected after $24 \mathrm{hr}$. (c) Plaque assay performed over 9 days. In contrast to the parental strain ( $\triangle K$ KU8), no lysis plaques are formed for TgDHHC2-iKD and TgDHHC14-iKD in presence of ATc, attesting for a defect in one or several steps of the lytic cycle in absence of the corresponding enzyme 
(a)

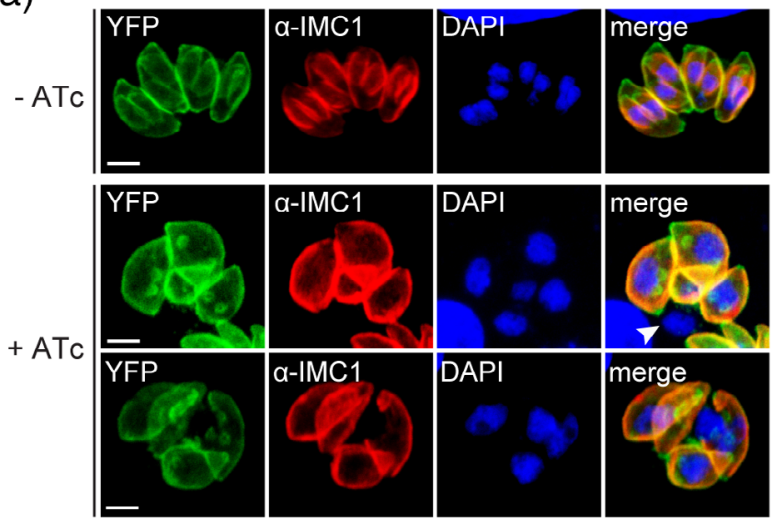

(b)

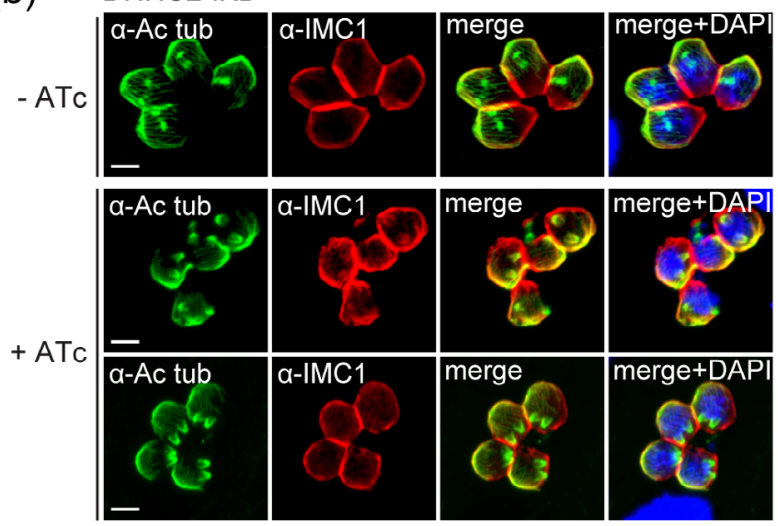

(c)
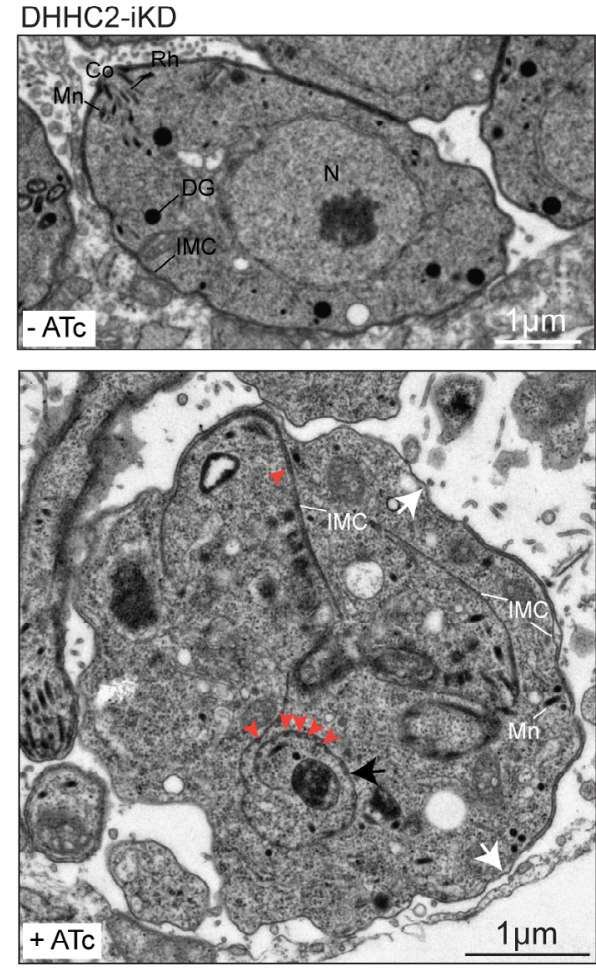

DHHC14-iKD / GAP50-YFP

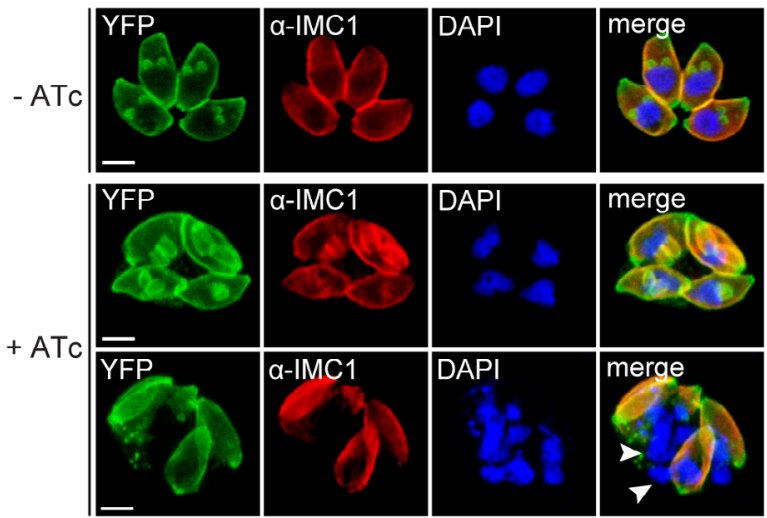

DHHC14-iKD
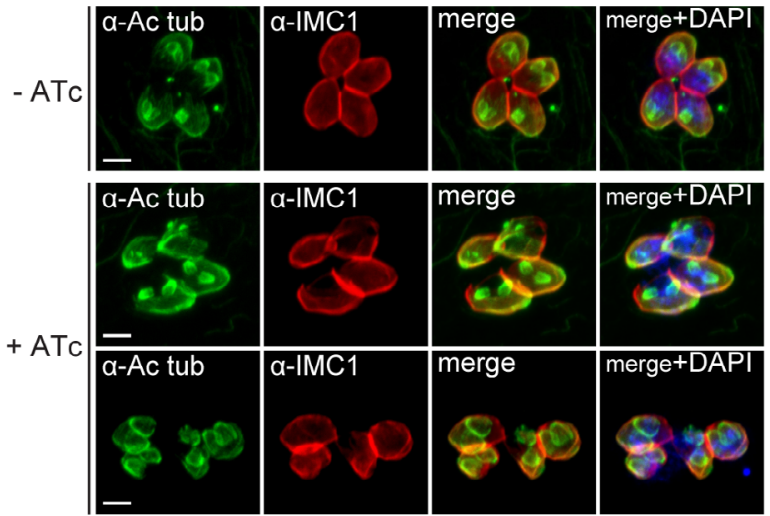

DHHC14-iKD
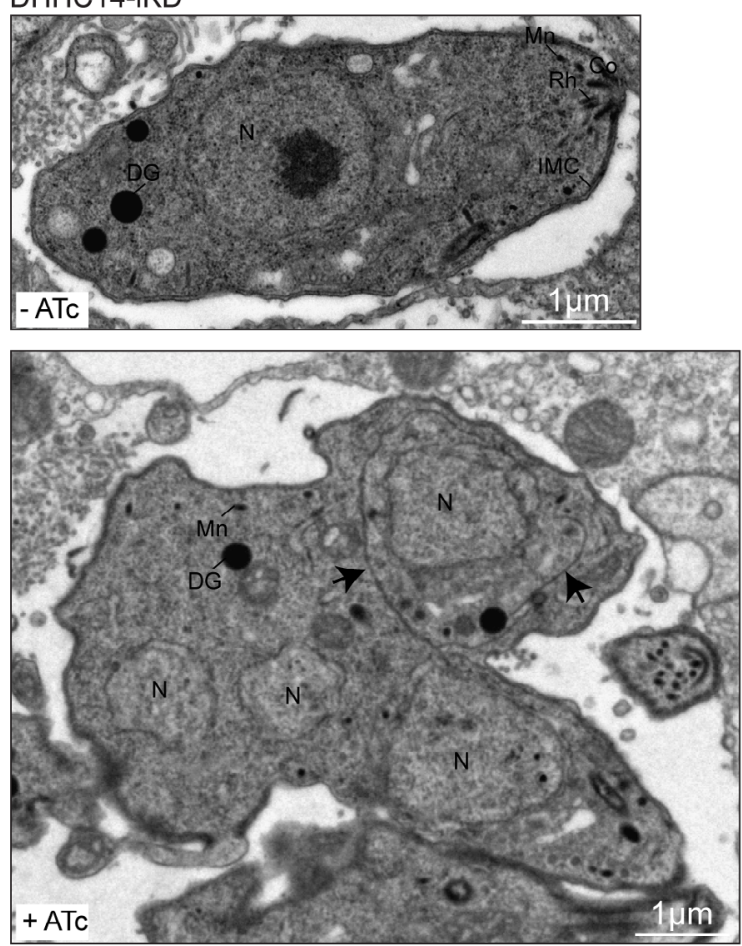

FIGURE 3 TgDHHC2 and TgDHHC14 are critical for the morphology of the parasites. TgDHHC2- and TgDHHC14-iKD tachyzoites expressing the endogenously tagged TgGAP50-YFP (a) or stained with the anti-acetylated tubulin antibodies (b) were treated for $16 \mathrm{hr} \pm$ ATc before fixation. TgIMC1 stains the alveolin network. Scale bars: $2 \mu \mathrm{m}$. (c) Electron microscopy pictures of TgDHHC2- and TgDHHC14-iKD tachyzoites treated \pm ATc for $24 \mathrm{hr}$. In TgDHHC2 depleted parasites, the IMC is not always associated to the plasma membrane of the mother cell (white arrows) and IMC material (black arrow) can be seen inside the parasite associated to microtubules (red arrowhead). Co, conoid; DG, dense granule; IMC, inner membrane complex; $\mathrm{Mn}$, microneme; $\mathrm{N}$, nucleus; $\mathrm{Rh}$, rhoptry 
collapse of the developing IMC occurs earlier upon the TgDHHC2 knock-down compare to TgDHHC14 indicating a sequential requirement of these enzymes to build the sub-compartments of the IMC.

The strong phenotype observed upon the depletion of TgDHHC2 and $\mathrm{TgDHHC} 14$ likely results from the simultaneous destabilisation of several palmitoylated proteins associated with the IMC. We have investigated few of them that have been shown experimentally to be palmitoylated and/or have been identified in the global palmitoylome of T. gondii (Beck et al., 2010; Chen et al., 2015, 2017; Foe et al., 2015). Among them, TgISP1-3 are dually acylated at their $\mathrm{N}$-terminus by myristoylation that occurs co-translationally on the $\mathrm{N}$-terminal glycine after cleavage of the methionine and by palmitoylation. The myristoylation can confer membrane association to the protein but is not strong enough to stabilise the protein into the membrane that therefore goes on and off the membrane (Peitzsch \& McLaughlin, 1993). Immunofluorescence assays combined with solubilisation experiments revealed that TgISP1 and TgISP3 are likely substrates of TgDHHC2 while no TgISPs seem to be impacted by TgDHHC14 depletion. A similar trend is observed for the palmitoylated proteins TgIAP1 and TgIAP2, upon the down-regulation of TgDHHC2, suggesting an enzyme-substrate relationship between these proteins. The proportion of the potential substrates still associated with the membranous structure upon enzyme depletion can be due to the presence of other acylation and/or the residual expression of the enzyme $16 \mathrm{hr}$ after the ATc treatment. Although TgISP1 and TgISP3 are not individually essential for the tachyzoite survival (Beck et al., 2010; Sidik et al., 2016), their combined destabilisation could account for the observed phenotype upon $\mathrm{TgDHHC} 2$ depletion. It is also legitimate to speculate that other substrates participate to this phenotype such as alveolins (TgIMCs) which compose the intermediate filamentous meshwork (Anderson-White et al., 2011) or the IMC transmembrane protein TgGAP40 for example. Indeed, our data are reminiscent of the TgGAP40-iKO phenotype (Harding et al., 2016) and TgGAP40 has been isolated in the palmitoylome of both $T$. gondii and $P$. falciparum (Foe et al., 2015; Jones, Collins, et al., 2012). The predicted palmitoylation site is on the cytoplasmic face just after the fourth transmembrane domain and could modulate the role of TgGAP40 in the biogenesis of the IMC. Further experiments will be needed to test this hypothesis.

While the spatial localisation of PATs confers their association with substrates, protein motifs in these enzymes are important for potentiating enzyme-substrate interactions as well. TgDHHC14 contains at its $\mathrm{N}$-terminus an ankyrin repeat domain, a 33-residue motif which mediates protein-protein interactions. The ankyrin repeat domain of human DHHC17, also known as huntingtin interacting proteins 14 (Hip14) has been shown to confer its palmitoylating activity on the huntingtin proteins, and also to transfer the substrate specificity when fused with another DHHC (Huang et al., 2009). Deletion of the ankyrin domain appears to affect the stability of TgDHHC14, leading to disappearance of the protein as evaluated by western blot. This domain likely plays a role in determining its localisation along the IMC sutures or in providing substrate specificity, thereby regulating the palmitoylating activity of TgDHHC14. Despite our attempts, none of the palmitoylated proteins tested seem to be affected by the depletion of TgDHHC14. The potential substrates likely reside in the transverse sutures of the IMC and only six proteins have been localised there so far (Chen et al., 2017; Lentini et al., 2015). Out of them, only TgTSC5, is predicted to affect the tachyzoite fitness upon depletion and is found in the T. gondii palmitoylome (Foe et al., 2015; Sidik et al., 2016). This protein is predicted to have three transmembrane domains but no palmitoylation site can be found in silico so further work will be needed to functionally characterise this candidate and establish if it is a substrate of TgDHHC14.

\section{4 | EXPERIMENTAL PROCEDURES}

\section{1 | Preparation of T. gondii genomic DNA and cDNA}

All genomic DNA (gDNA) have been prepared from tachyzoites (RH strain) using the Wizard SV genomic DNA purification system (Promega).

\subsection{Cloning of DNA constructs}

All amplifications for cloning were performed with the Q5 polymerase (New England Biolabs) and the primers used are listed in Table S1 in Data S1. All amplifications for screening were performed with the GoTaq DNA polymerase (Promega) and the primers listed in Table S2 in Data S1.

\subsection{1 | Tet-inducible knockdown of TgDHHC2 (DHHC2-iKD)}

A $586 \mathrm{bp}$ gDNA fragment of the $\mathrm{N}$-terminus of $\mathrm{TgDHHC2}$ (TGME49_278850) was amplified by PCR with primers TgDHHC2-5174/TgDHHC2-5175, digested with Avrll and Spel restriction enzymes and cloned into a modified version of the $5^{\prime} \mathrm{COR}$ TATi1-HX-tetO7S1MycCOR vector (Salamun, Kallio, Daher, SoldatiFavre, \& Kursula, 2014). Then, a 535 bp gDNA fragment of the $5^{\prime}$ flanking region of $\mathrm{TgDHHC2}$ was amplified using primers TgDHHC2-5172/TgDHHC2-5173 and cloned into the Ncol and Nhel sites. To direct the insertion of the plasmid digested with Avrll and Nhel before transfection, a specific gRNA vector has been generated using the Q5 site-directed mutagenesis kit (New England Biolabs) with the vector pSAG1::CAS9-GFP-U6::sgUPRT as template (Shen, Brown, Lee, \& Sibley, 2014) and the primer pair TgDHHC2-5171/gRNA-4883.

\subsection{2 | Tet-inducible knockdown of TgDHHC14 (DHHC14-iKD)}

A PCR fragment encoding the TATi trans-activator, the HXGPRT cassette and the TetO7S1 promoter was generated using the 
KOD DNA polymerase (Novagen, Merk) with the vector $5^{\prime}$ CORTATi1-HX-tetO7S1MycCOR as template and the primers TgDHHC14-5362 and TgDHHC14-5363 that also carry $30 \mathrm{bp}$ homology with the $5^{\prime}$ end of $\mathrm{TgDHHC14}$. To direct the insertion of the PCR product at the start of TgDHHC14 (TGME49_293730), a specific sgRNA vector has been generated as described above using the primer pair TgDHHC14-5181/ gRNA-4883.

\subsection{3 | C-terminal tagging of TgDHHC2 and TgDHHC14}

To endogenously tag TgDHHC2 and TgDHHC14, the HXGPRT cassette of the KI-DHHC2-3Ty-HX and KI-DHHC2-3Ty-HX vectors (Frénal et al., 2013) has been replaced by the DHFR-TS cassette subcloned into the two Sacll sites.

\subsection{4 | Complementations of DHHC2-iKD and DHHC14-iKD}

To complement the DHHC2-iKD and DHHC14-iKD with a wild type copy and a dead enzyme mutant, the UPRT-pRON5-G13-4myc vector (Guérin et al., 2017) has been used to subclone the endogenous promoter and the coding sequence of each gene. For $\mathrm{DHHC2}$, the promoter was amplified from gDNA using the primer pair TgDHHC2-8626/TgDHHC2-8627 and cloned between the Notl and $\mathrm{Pacl}$ sites. Then the coding sequence amplified from cDNA with the primers TgDHHC2-8624/TgDHHC2-8625 was cloned between the $\mathrm{Pacl}$ and EcoRV sites. To generate the DHHC2-C194S mutant, the previous UPRT-pDHHC2-DHHC2-4myc vector was used as template for Q5 site directed mutagenesis using the primer pair TgDHHC2-8707/ TgDHHC2-8708. For DHHC14, the intermediate vector 5'DHHC14-pDHHC14-loxP-DHHC14-3Ty-loxP-YFP-3'DHHC14 vector was used as a template to amplify pDHHC14-loxP-DHHC14 with the primers TgDHHC14-8628/TgDHHC14-8629. The fragment was assembled into the UPRT-pRON5-G13-4myc vector digested with Notl and EcoRV using the NEBuilder HiFi DNA Assembly kit to generate the UPRT-pDHHC14-DHHC14-4myc vector. This last vector was then used as template for Q5 site directed mutagenesis using the primer pair TgDHHC14-8703/TgDHHC14-8704 to generate the DHHC14-C618S mutant and using the primers TgDHHC14-8705/ TgDHHC14-8706 to generate the DHHC14- $\triangle \mathrm{ANK}$ mutant.

\subsection{5 | pGAP50-YFP-CAT}

The $3^{\prime}$ end of the TgGAP50 gene (gDNA, TGME49_219320) was amplified using the primers TgGAP50-2900/TgGAP50-2901 and then cloned in frame with the YFP in the pYFP-LIC-CAT vector as previously described using the ligation independent cloning (LIC) technology (Huynh \& Carruthers, 2009).

\subsection{6 | pKI-IAP1-Myc}

A gDNA fragment corresponding to the $3^{\prime}$ end of TgIAP1 (TGME49_283510) was amplified using the primers pair TgIAP1-3631/TgIAP1-3617 and cloned in the vector pT8-ARO-MycBleo (Mueller et al., 2013) between the Kpnl and Nsil sites.

\subsection{7 | pKI-IAP2-2Ty}

To tag TgIAP2 (TGME49_297130) at its C-terminal extremity, a PCR fragment containing $32 \mathrm{bp}$ homology with the $5^{\prime}$ end of TgIAP2 has been amplified using the vector pLinker-2xTy-DHFR-LoxP as template and the primers pair TgIAP2_8120/TgIAP2_8121. To direct the insertion of the PCR product at the $5^{\prime}$ end of IAP2, a specific sgRNA vector has been generated as described above using the primer pair TgIAP1-8122/gRNA-4883.

\section{3 | Toxoplasma gondii tachyzoite cultures}

T. gondii tachyzoites strains were grown in confluent human foreskin fibroblasts (HFF) maintained in Dulbecco's Modified Eagle's Medium (Gibco DMEM) supplemented with $5 \%$ fetal calf serum, $2 \mathrm{mM}$ glutamine and $25 \mu \mathrm{g} / \mathrm{mL}$ gentamicin. Conditional expression of the Tetinducible constructs was performed using $1 \mu \mathrm{g} / \mathrm{ml}$ anhydrotetracycline (ATc) (Meissner, Schlüter, \& Soldati, 2002).

\subsection{Parasite transfection and selection of stable transformants}

Parasite transfections were performed as previously described (Soldati \& Boothroyd, 1993). RH $\Delta$ KU80 (Huynh \& Carruthers, 2009) and derivative strains have been transfected with 15-20 $\mu \mathrm{g}$ of the linearised knock-in constructs. Either mycophenolic acid (MPA, $25 \mathrm{mg} / \mathrm{ml})$ and xanthine $(50 \mathrm{mg} / \mathrm{ml})$ or pyrimethamine $(1 \mu \mathrm{g} / \mathrm{ml})$ was used to select the resistant parasites carrying the HXGPRT or the DHFR cassette respectively. For the CRISPR-Cas9 strategy, $15 \mu \mathrm{g}$ of the circular gRNA-specific CRISPR/CAS9 vector was transfected into $\mathrm{RH} \triangle \mathrm{KU} 80$ together with the product of $2 \mathrm{PCR}$ reactions. For complementation, $40 \mu \mathrm{g}$ of the complemented vectors, digested by Ndel for DHHC14 vectors and by Apal and BamHI for DHHC2 plasmids, were transfected with $15 \mu \mathrm{g}$ of the UPRT-specific sgRNA plasmid pU6-UPRT (Guérin et al., 2017), a gift from Dr Maryse Lebrun.

\section{5 $\quad$ Antibodies}

The antibodies used in this study were previously described as follow: polyclonal rabbit: $\alpha$-IMC1 (1:1000 WB and IFA) and $\alpha$-GAP45 (1:10000 WB and IFA) (Frénal et al., 2014), $\alpha$-Catalase (1:2000 WB) (Ding, Clayton, \& Soldati, 2000) and $\alpha$-PRF (1:2000 WB, [Plattner 
(a)

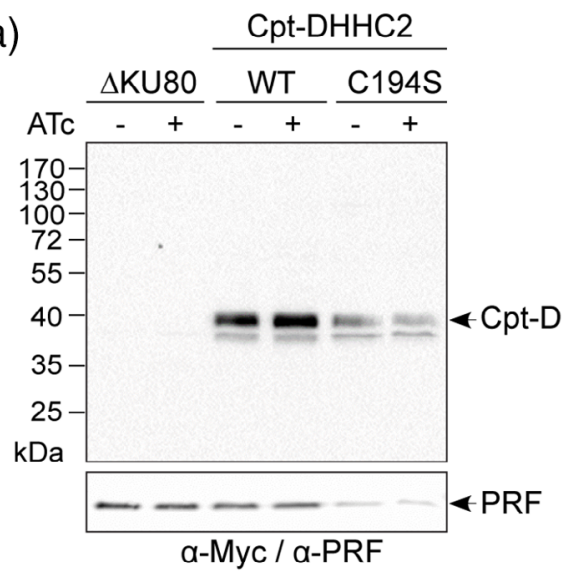

(b) Cpt-DHHC2-WT-4Myc

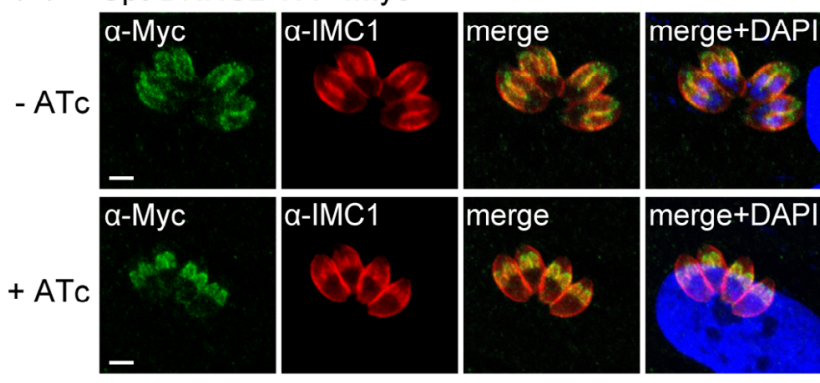

Cpt-DHHC2-C194S-4Myc
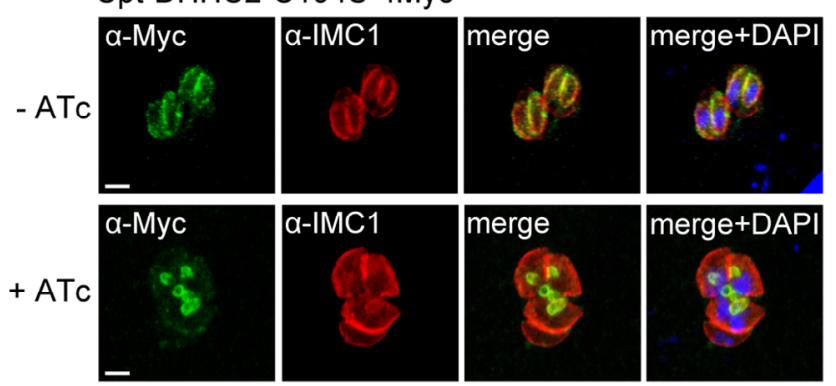

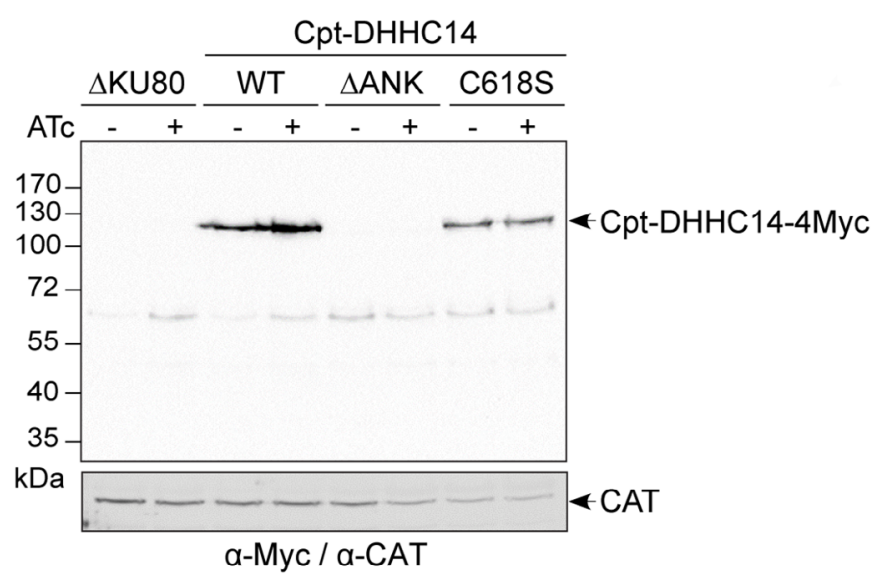

(c)
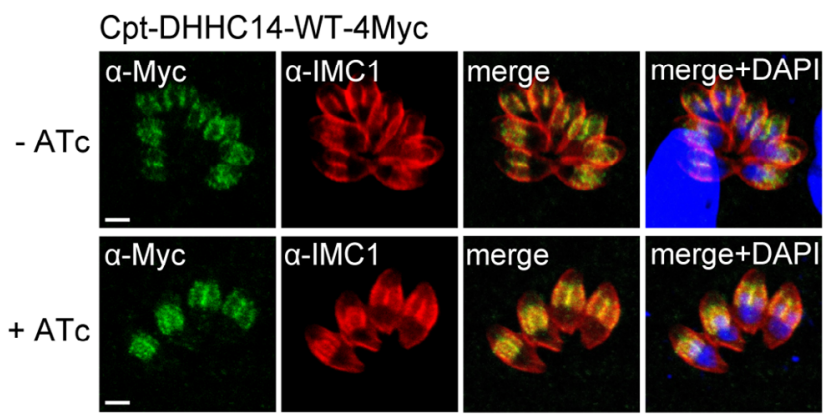

\section{Cpt-DHHC14-C618S-4Myc}
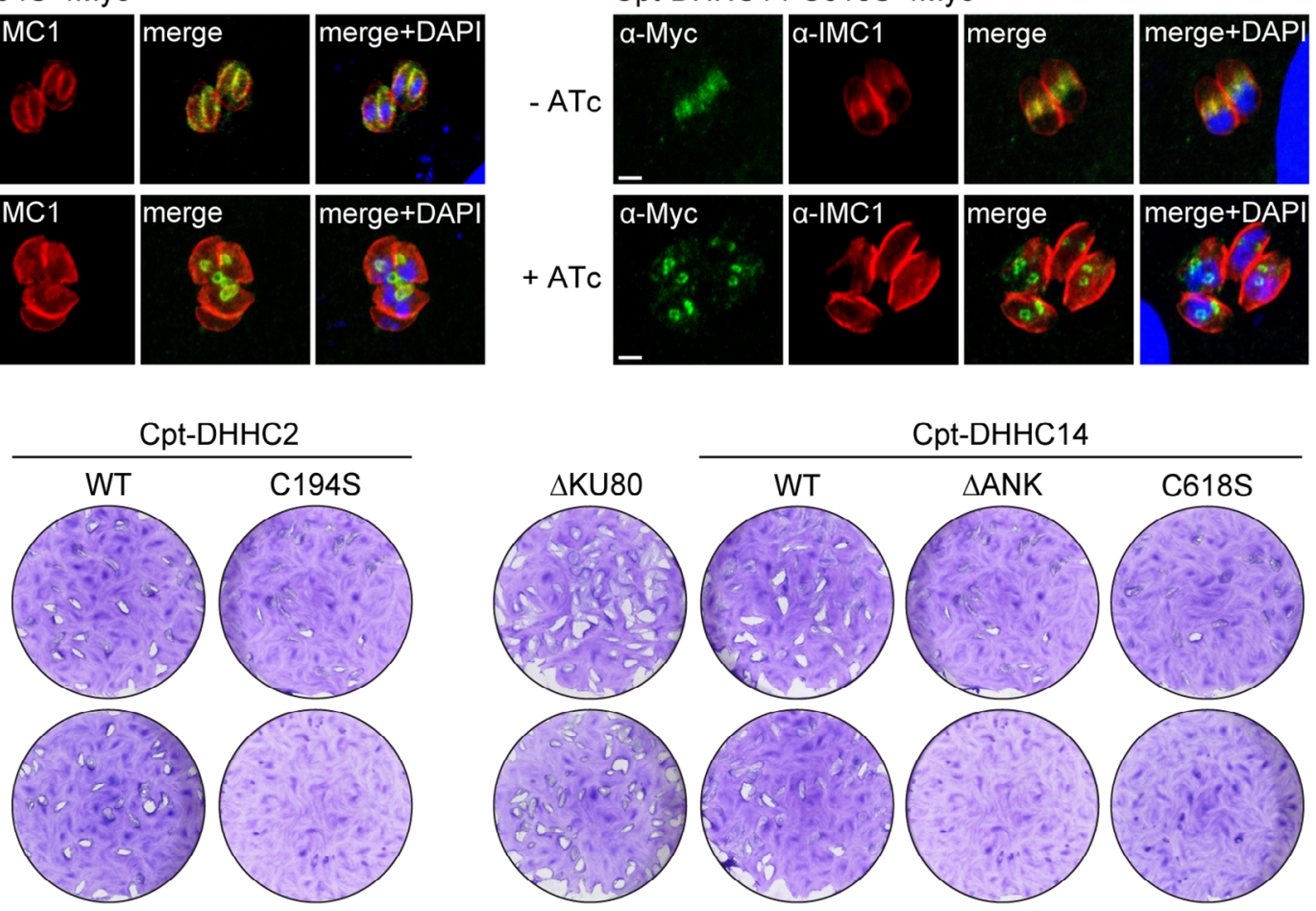

FIGURE 4 The activity of TgDHHC2 and TgDHHC14 is critical for the IMC biogenesis. (a) TgDHHC2-iKD and TgDHHC14-iKD strains, complemented with either the wild type (cpt-DHHC2/14-WT) or the mutants (cpt-DHHC2-C194S, cpt-DHHC14-C618S, cpt-DHHC- $\triangle$ ANK) were treated for $24 \mathrm{hr} \pm$ ATc and analysed by western blot. Catalase (CAT)/Profilin (PRF) was used as loading control. (b) The same strains were also analysed by IFA. Scale bars: $2 \mu \mathrm{m}$. (c) The drastic impairment of the lytic cycle seen in TgDHHC2-iKD and TgDHHC14-iKD strains, as assessed by plaque formation after 7 days in the presence of ATc, is rescued by complementation with the wild type DHHCs but not the acyltransferase mutants 

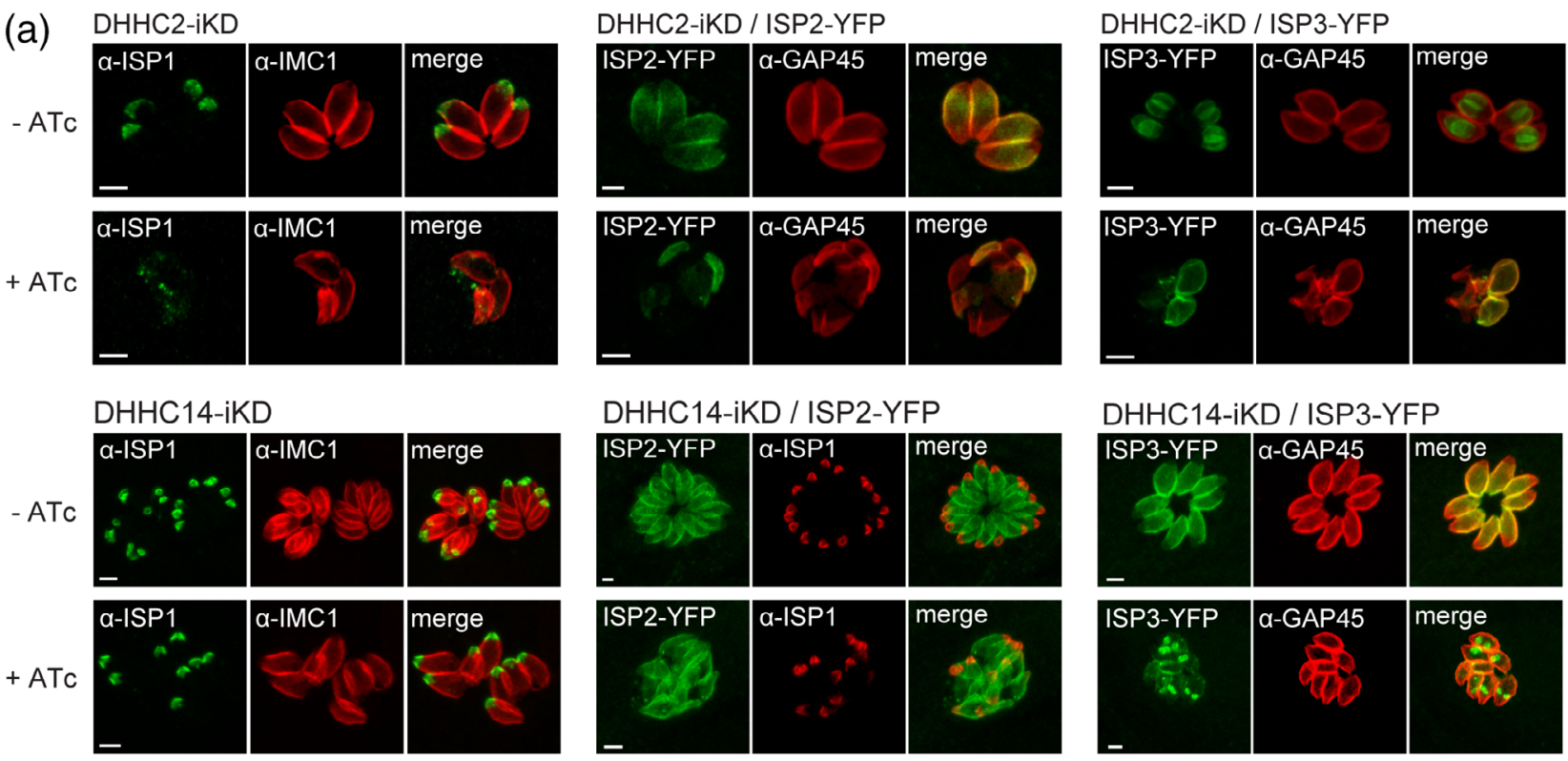

(b)

DHHC2-iKD
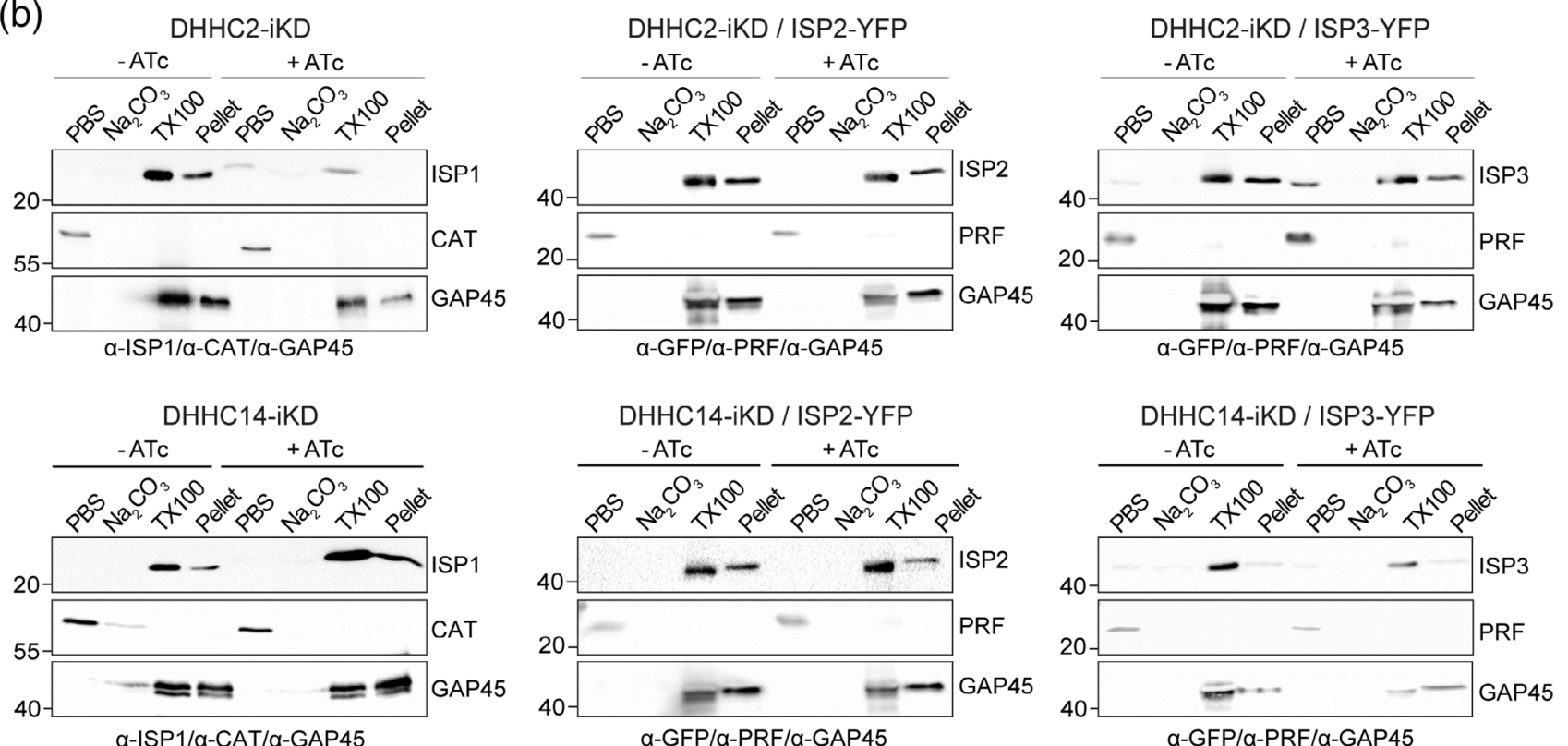

FIGURE 5 Evaluation of TgISPs as substrates of TgDHHC2 and/or TgDHHC14. (a) The localisation of the IMC sub-compartment proteins (TgISPs) was evaluated in TgDHHC2-iKD and TgDHHC14-iKD strains, following treatment for $16 \mathrm{hr} \pm$ ATc and fixation. TgISP1 localisation was assessed using specific antibodies while TgISP2 and TgISP3 were endogenously tagged at the C-terminus with YFP. TgIMC1 stains the alveolin network, and TgGAP45 stains the pellicle. Scale bars: $2 \mu \mathrm{m}$. (b) The solubility of TgISPs in the TgDHHC2-iKD and TgDHHC14-iKD, treated for $16 \mathrm{hr} \pm$ ATc, was analysed by western blot after serial fractionation assay. TgISPs were detected using anti-TgISP1 or anti-GFP antibodies. The cytosolic profilin (TgPRF) and the pellicular TgGAP45 were used as controls of solubilisation

et al., 2008]); monoclonal mouse, $\alpha$-ACT (supernatant of hybridoma, 1:10 WB [Herm-Götz et al., 2002]), $\alpha$-ISP1 (1:2000 WB and IFA, (Beck et al., 2010)), $\alpha$-Myc (9E10, supernatant of hybridoma, 1:10 WB and IFA), $\alpha$-Ty (BB2, supernatant of hybridoma, 1:10 WB and IFA [Bastin, Bagherzadeh, Matthews, \& Gull, 1996]), monoclonal rat $\alpha-\mathrm{HA}$ (1:1000 IFA, Roche), $\alpha$-acetylated $\alpha$-tubulin (6-11B-1, 1:1000 IFA, Abcam). For western blot analyses, secondary peroxidase conjugated goat $\alpha$-rabbit/mouse antibodies (Sigma) were used. For immunofluorescence analyses, the secondary antibodies Alexa Fluor 488- and
Alexa Fluor 594-conjugated goat $\alpha$-mouse/rabbit antibodies (1:3000 IFA, Thermofisher) were used.

\subsection{Immunofluorescence assay (IFA)}

Parasite-infected HFF cells seeded on cover slips were fixed with $4 \%$ PFA/0.05\% glutaraldehyde (PFA/GA) in PBS for 10 min or with methanol for $8 \mathrm{~min}$ for anti-tubulin-Ac and then processed as previously described 
(a) DHHC2-iKD / IAP1-myc
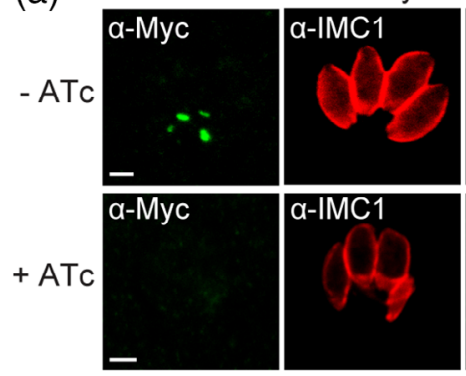

DHHC14-iKD / IAP1-myc
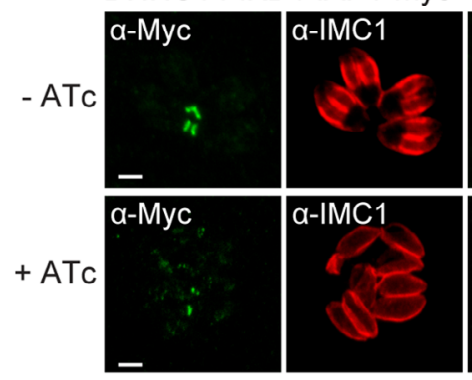

(b)
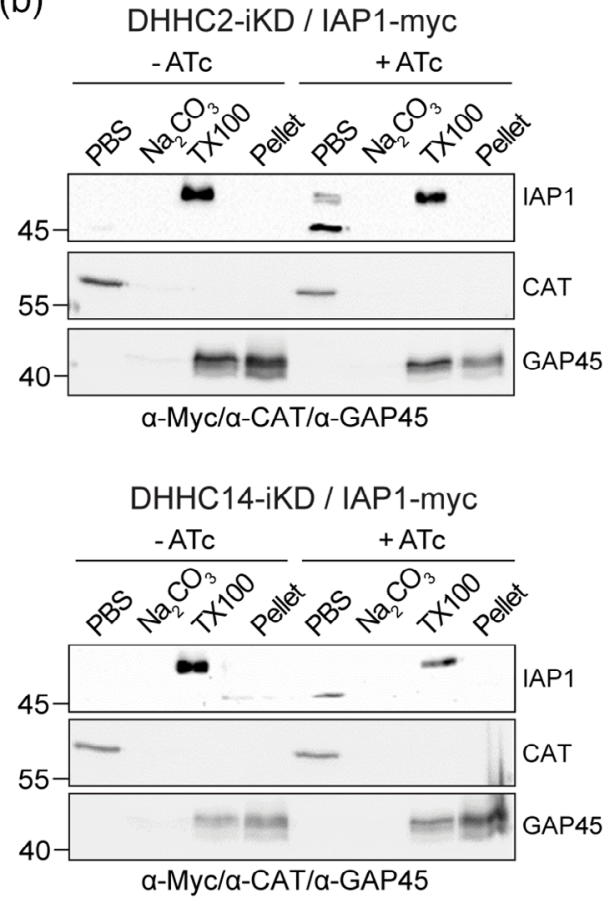
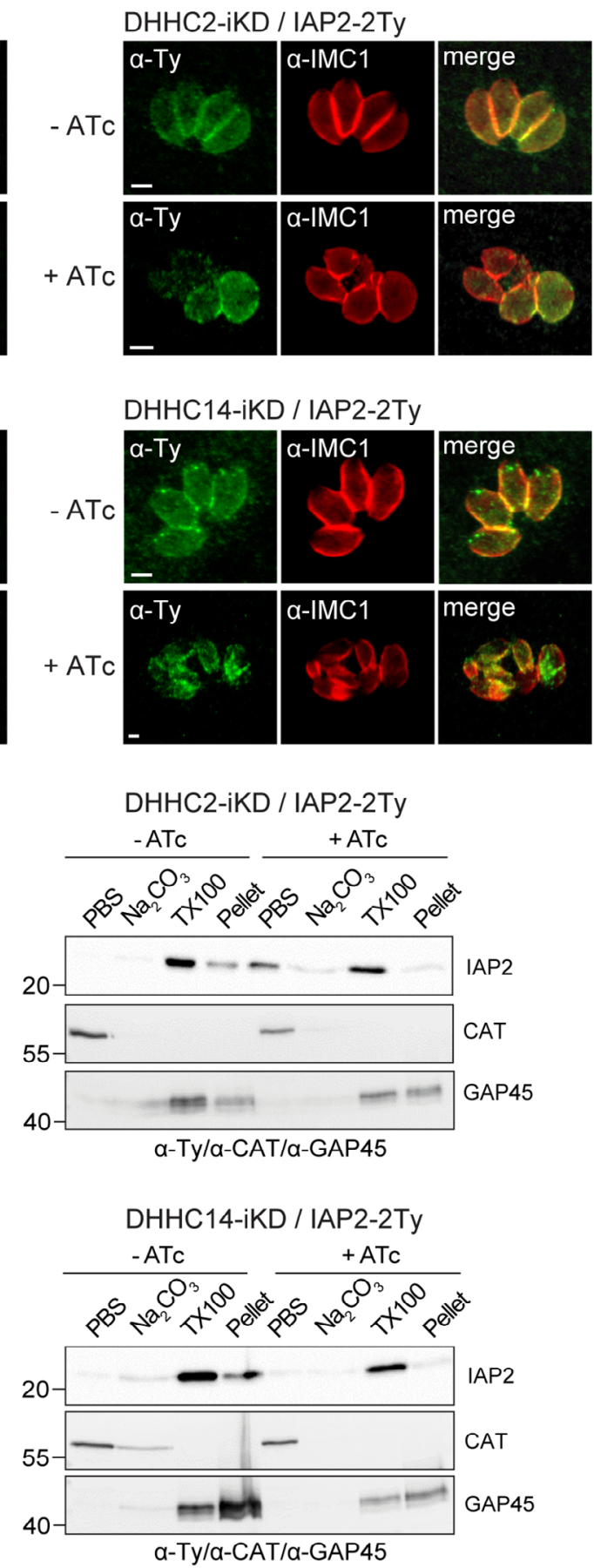

FIGURE 6 Evaluation of

TgIAP1/2 as substrates of TgDHHC2 and/or TgDHHC14.

(a) The localisations of the IMC associated proteins (TgIAPs) were evaluated in TgDHHC2-iKD and TgDHHC14-iKD strains, following treatment for $16 \mathrm{hr} \pm \mathrm{ATC}$ and fixation. TgIMC1 stains the alveolin network. TgIAP1 and TgIAP2 were endogenously tagged at the C-terminus with $4 \mathrm{c}-\mathrm{Myc}$ or 3 Ty tags, respectively. (b) The solubility of TgIAPs in the TgDHHC2-iKD and TgDHHC14-iKD, treated for $16 \mathrm{hr} \pm \mathrm{ATc}$, was analysed by western blot after serial fractionation assay using antiMyc and anti-Ty antibodies. TgPRF and TgGAP45 were used as controls for the cytosolic and the cytoskeletal fractions, respectively
(Hettmann et al., 2000). Confocal images were generated with a Zeiss LSM700 laser scanning confocal microscope using an apochromat $63 x$ /1.4 oil objective at the Bioimaging core facility of the Faculty of Medicine, University of Geneva. Stacks of sections were processed with ImageJ and projected using the maximum projection tool.

\section{7 | Western blot analysis}

Parasites were lysed in RIPA buffer (150 mM NaCl, 1\% Triton X-100, $0.5 \%$ deoxycholate, $0.1 \% \mathrm{SDS}, 50 \mathrm{mM}$ Tris $\mathrm{pH} 7.5$ ), incubated on ice for $15 \mathrm{~min}$ and then centrifuge for $30 \mathrm{~min}$ at $14,000 \mathrm{rpm}$ at $4^{\circ} \mathrm{C}$. The supernatant was then collected and mixed with SDS-PAGE loading buffer under reducing conditions. Separated proteins were transferred to nitrocellulose membranes and probed with appropriate antibodies in $5 \%$ non-fat milk powder in PBS-0.05\% Tween20. Bound secondary peroxidase conjugated antibodies were visualised using the ECL system.

\section{8 | Plaque assay}

Confluent HFF cells were infected with freshly egressed parasites and treated \pm ATc if necessary. After 7 days, the cells were fixed with PFA/GA and stained with a crystal violet solution (Sigma). 


\section{9 | Transmission electron microscopy}

Freshly egressed parasites were inoculated on confluent HFFs and allowed to grow for $24 \mathrm{hr} \pm$ ATc Infected host cells were washed with $0.1 \mathrm{M}$ phosphate buffer $\mathrm{pH} 7.4$, then fixed with $2.5 \%$ glutaraldehyde in $0.1 \mathrm{M}$ phosphate buffer $\mathrm{pH} 7.4$, scrapped and pelleted. Samples were then treated as previously described (Frénal et al., 2010). Thin sections were analysed using a Technai 20 electron microscope (FEI Company) at the "Pôle Facultaire de Microscopie Ultrastructurale (PFMU)" of the Faculty of medicine of Geneva.

\section{ACKNOWLEDGEMENTS}

We gratefully acknowledge Prof Dominique Soldati-Favre for her the assistance and support and Jean-Baptiste Marq for the documentation of EM pictures. We also thanks Dr. David Sibley (Washington University) for providing the CRISPR/Cas9 and pLinker-2xTy-DHFRLoxP plasmids, Dr Vernon Carruthers (University of Michigan) for the LIC vector, Dr Maryse Lebrun (University of Montpellier) for the ISP2-YFP, ISP3-YFP, SIP-HA and UPRT vectors and Dr Christina Mueller for the super-resolution image of DHHC14-3Ty by SIM. This work was supported by the "Sir Jules Thorn Charitable Overseas Trust reg., Schaan" 2014 grant for young researchers to KF. SKD was supported by the "Sir Jules Thorn Charitable Overseas Trust reg., Schaan" 2014 grant (to KF) and by MalarX (ME9782 to DSF).

\section{CONFLICT OF INTEREST}

The authors declare no conflict of interest.

\section{ORCID}

Karine Frénal (DD https://orcid.org/0000-0002-8952-2598

\section{REFERENCES}

Anderson-White, B., Beck, J. R., Chen, C.-T., Meissner, M., Bradley, P. J., \& Gubbels, M.-J. (2012). Cytoskeleton assembly in Toxoplasma gondii cell division. International Review of Cell and Molecular Biology, 298, 1-31.

Anderson-White, B. R., Ivey, F. D., Cheng, K., Szatanek, T., Lorestani, A., Beckers, C. J., ... Gubbels, M. J. (2011). A family of intermediate filament-like proteins is sequentially assembled into the cytoskeleton of Toxoplasma gondii. Cellular Microbiology, 13, 18-31.

Bastin, P., Bagherzadeh, Z., Matthews, K. R., \& Gull, K. (1996). A novel epitope tag system to study protein targeting and organelle biogenesis in Trypanosoma brucei. Molecular and Biochemical Parasitology, 77, 235-239.

Beck, J. R., Fung, C., Straub, K. W., Coppens, I., Vashisht, A. A., Wohlschlegel, J. A., \& Bradley, P. J. (2013). A Toxoplasma palmitoyl acyl transferase and the palmitoylated armadillo repeat protein TgARO govern apical rhoptry tethering and reveal a critical role for the rhoptries in host cell invasion but not egress. PLoS Pathogens, 9, e1003162.

Beck, J. R., Rodriguez-Fernandez, I. A., de Leon, J. C., Huynh, M.-H., Carruthers, V. B., Morrissette, N. S., \& Bradley, P. J. (2010). A novel family of Toxoplasma IMC proteins displays a hierarchical organization and functions in coordinating parasite division. PLoS Pathogens, 6, e1001094.

Blanc, M., David, F., Abrami, L., Migliozzi, D., Armand, F., Bürgi, J., \& van der Goot, F. G. (2015). SwissPalm: Protein palmitoylation database. F1000Research, 4, 261.
Blaskovic, S., Blanc, M., \& van der Goot, F. G. (2013). What does Spalmitoylation do to membrane proteins? The FEBS Journal, 280, 2766-2774.

Bullen, H. E., Tonkin, C. J., O'Donnell, R. A., Tham, W.-H., Papenfuss, A. T., Gould, S., ... Gilson, P. R. (2009). A novel family of apicomplexan glideosome-associated proteins with an inner membrane-anchoring role. The Journal of Biological Chemistry, 284, 25353-25363.

Caballero, M. C., Alonso, A. M., Deng, B., Attias, M., de Souza, W., \& Corvi, M. M. (2016). Identification of new palmitoylated proteins in Toxoplasma gondii. Biochimica et Biophysica Acta, 1864, 400-408.

Chen, A. L., Kim, E. W., Toh, J. Y., Vashisht, A. A., Rashoff, A. Q., Van, C., ... Bradley, P. J. (2015). Novel components of the Toxoplasma inner membrane complex revealed by BiolD. mBio, 6, e02357.

Chen, A. L., Moon, A. S., Bell, H. N., Huang, A. S., Vashisht, A. A., Toh, J. Y., ... Bradley, P. J. (2017). Novel insights into the composition and function of the Toxoplasma IMC sutures. Cellular Microbiology, 19, e12678.

Ding, M., Clayton, C., \& Soldati, D. (2000). Toxoplasma gondii catalase: Are there peroxisomes in toxoplasma? Journal of Cell Science, 113(Pt 13), 2409-2419.

Dubremetz, J. F., \& Torpier, G. (1978). Freeze fracture study of the pellicle of an eimerian sporozoite (Protozoa, Coccidia). Journal of Ultrastructure Research, 62, 94-109.

Emmer, B. T., Nakayasu, E. S., Souther, C., Choi, H., Sobreira, T. J. P., Epting, C. L., ... Engman, D. M. (2011). Global analysis of protein palmitoylation in African trypanosomes. Eukaryotic Cell, 10, 455-463.

Foe, I. T., Child, M. A., Majmudar, J. D., Krishnamurthy, S., van der Linden, W. A., Ward, G. E., ... Bogyo, M. (2015). Global analysis of palmitoylated proteins in Toxoplasma gondii. Cell Host \& Microbe, 18, 501-511.

Frénal, K., Marq, J.-B., Jacot, D., Polonais, V., \& Soldati-Favre, D. (2014). Plasticity between MyoC- and MyoA-glideosomes: An example of functional compensation in Toxoplasma gondii invasion. PLoS Pathogens, 10, e1004504.

Frénal, K., Polonais, V., Marq, J.-B., Stratmann, R., Limenitakis, J., \& SoldatiFavre, D. (2010). Functional dissection of the apicomplexan glideosome molecular architecture. Cell Host \& Microbe, 8, 343-357.

Frénal, K., Tay, C. L., Mueller, C., Bushell, E. S., Jia, Y., Graindorge, A., ... Soldati-Favre, D. (2013). Global analysis of apicomplexan protein Sacyl transferases reveals an enzyme essential for invasion. Traffic, 14, 895-911.

Fung, C., Beck, J. R., Robertson, S. D., Gubbels, M.-J., \& Bradley, P. J. (2012). Toxoplasma ISP4 is a central IMC sub-compartment protein whose localization depends on palmitoylation but not myristoylation. Molecular and Biochemical Parasitology, 184, 99-108.

Gilk, S. D., Raviv, Y., Hu, K., Murray, J. M., Beckers, C. J. M., \& Ward, G. E. (2006). Identification of PhIL1, a novel cytoskeletal protein of the Toxoplasma gondii pellicle, through photosensitized labeling with 5-[ $\left.\left.{ }^{125}\right]\right]$ iodonaphthalene-1-azide. Eukaryotic Cell, 5, $1622-1634$

Guérin, A., Corrales, R. M., Parker, M. L., Lamarque, M. H., Jacot, D., El Hajj, H., ... Lebrun, M. (2017). Efficient invasion by Toxoplasma depends on the subversion of host protein networks. Nature Microbiology, 2, 1358-1366.

Harding, C. R., Egarter, S., Gow, M., Jiménez-Ruiz, E., Ferguson, D. J. P., \& Meissner, M. (2016). Gliding associated proteins play essential roles during the formation of the inner membrane complex of Toxoplasma gondii. PLoS Pathogens, 12, e1005403.

Harding, C. R., Gow, M., Kang, J. H., Shortt, E., Manalis, S. R., Meissner, M., \& Lourido, S. (2019). Alveolar proteins stabilize cortical microtubules in Toxoplasma gondii. Nature Communications, 10, 401.

Herm-Götz, A., Weiss, S., Stratmann, R., Fujita-Becker, S., Ruff, C., Meyhöfer, E., ... Soldati, D. (2002). Toxoplasma gondii myosin A and its light chain: A fast, single-headed, plus-end-directed motor. The EMBO Journal, 21, 2149-2158. 
Hettmann, C., Herm, A., Geiter, A., Frank, B., Schwarz, E., Soldati, T., \& Soldati, D. (2000). A dibasic motif in the tail of a class XIV apicomplexan myosin is an essential determinant of plasma membrane localization. Molecular Biology of the Cell, 11, 1385-1400.

Huang, K., Sanders, S., Singaraja, R., Orban, P., Cijsouw, T., Arstikaitis, P., ... El-Husseini, A. (2009). Neuronal palmitoyl acyl transferases exhibit distinct substrate specificity. The FASEB Journal, 23(8), 2605-2615.

Huynh, M.-H., \& Carruthers, V. B. (2009). Tagging of endogenous genes in a Toxoplasma gondii strain lacking Ku80. Eukaryotic Cell, 8, 530-539.

Jones, M. L., Collins, M. O., Goulding, D., Choudhary, J. S., \& Rayner, J. C. (2012). Analysis of protein palmitoylation reveals a pervasive role in Plasmodium development and pathogenesis. Cell Host \& Microbe, 12, 246-258.

Jones, M. L., Tay, C. L., \& Rayner, J. C. (2012). Getting stuck in: Protein palmitoylation in Plasmodium. Trends in Parasitology, 28, 496-503.

Lentini, G., Kong-Hap, M., El Hajj, H., Francia, M., Claudet, C., Striepen, B., ... Lebrun, M. (2015). Identification and characterization of Toxoplasma SIP, a conserved apicomplexan cytoskeleton protein involved in maintaining the shape, motility and virulence of the parasite. Cellular Microbiology, 17, 62-78.

Linder, M. E., \& Deschenes, R. J. (2007). Palmitoylation: Policing protein stability and traffic. Nature Reviews. Molecular Cell Biology, 8, 74-84.

Lobo, S., Greentree, W. K., Linder, M. E., \& Deschenes, R. J. (2002). Identification of a Ras palmitoyltransferase in Saccharomyces cerevisiae. The Journal of Biological Chemistry, 277, 41268-41273.

Long, S., Anthony, B., Drewry, L. L., \& Sibley, L. D. (2017). A conserved ankyrin repeat-containing protein regulates conoid stability, motility and cell invasion in Toxoplasma gondii. Nature Communications, 8, 2236.

Long, S., Brown, K. M., Drewry, L. L., Anthony, B., Phan, I. Q. H., \& Sibley, L. D. (2017). Calmodulin-like proteins localized to the conoid regulate motility and cell invasion by Toxoplasma gondii. PLoS Pathogens, 13, e1006379.

Mann, T., \& Beckers, C. (2001). Characterization of the subpellicular network, a filamentous membrane skeletal component in the parasite Toxoplasma gondii. Molecular and Biochemical Parasitology, 115, 257-268.

McCoy, J. M., Stewart, R. J., Uboldi, A. D., Li, D., Schröder, J., Scott, N. E., ... Tonkin, C. J. (2017). A forward genetic screen identifies a negative regulator of rapid $\mathrm{Ca}^{2+}$-dependent cell egress (MS1) in the intracellular parasite Toxoplasma gondii. The Journal of Biological Chemistry, 292, 7662-7674.

Meissner, M., Schlüter, D., \& Soldati, D. (2002). Role of Toxoplasma gondii myosin A in powering parasite gliding and host cell invasion. Science, 298, 837-840.

Mueller, C., Klages, N., Jacot, D., Santos, J. M., Cabrera, A., Gilberger, T. W., ... Soldati-Favre, D. (2013). The Toxoplasma protein ARO mediates the apical positioning of rhoptry organelles, a prerequisite for host cell invasion. Cell Host \& Microbe, 13, 289-301.

Nishi, M., Hu, K., Murray, J. M., \& Roos, D. S. (2008). Organellar dynamics during the cell cycle of Toxoplasma gondii. Journal of Cell Science, 121, 1559-1568.

Ovciarikova, J., Lemgruber, L., Stilger, K. L., Sullivan, W. J., \& Sheiner, L. (2017). Mitochondrial behaviour throughout the lytic cycle of Toxoplasma gondii. Scientific Reports, 16(7), 42746.

Peitzsch, R. M., \& McLaughlin, S. (1993). Binding of acylated peptides and fatty acids to phospholipid vesicles: Pertinence to myristoylated proteins. Biochemistry, 32, 10436-10443.

Plattner, F., Yarovinsky, F., Romero, S., Didry, D., Carlier, M.-F., Sher, A., \& Soldati-Favre, D. (2008). Toxoplasma profilin is essential for host cell invasion and TLR11-dependent induction of an interleukin-12 response. Cell Host \& Microbe, 3, 77-87.
Porchet, E., \& Torpier, G. (1977). Freeze fracture study of Toxoplasma and Sarcocystis infective stages (Author's transl). Zeitschrift für Parasitenkunde, 54, 101-124.

Rana, M. S., Kumar, P., Lee, C.-J., Verardi, R., Rajashankar, K. R., \& Banerjee, A. (2018). Fatty acyl recognition and transfer by an integral membrane S-acyltransferase. Science, 359, eaao6326.

Roth, A. F., Wan, J., Bailey, A. O., Sun, B., Kuchar, J. A., Green, W. N., ... Davis, N. G. (2006). Global analysis of protein palmitoylation in yeast. Cell, 125, 1003-1013.

Salamun, J., Kallio, J. P., Daher, W., Soldati-Favre, D., \& Kursula, I. (2014). Structure of Toxoplasma gondii coronin, an actin-binding protein that relocalizes to the posterior pole of invasive parasites and contributes to invasion and egress. The FASEB Journal, 28, 4729-4747.

Sangaré, L. O., Alayi, T. D., Westermann, B., Hovasse, A., Sindikubwabo, F., Callebaut, I., ... Tomavo, S. (2016). Unconventional endosome-like compartment and retromer complex in Toxoplasma gondii govern parasite integrity and host infection. Nature Communications, 7, 11191.

Santos, J. M., Kehrer, J., Franke-Fayard, B., Frischknecht, F., Janse, C. J., \& Mair, G. R. (2015). The Plasmodium palmitoyl-S-acyl-transferase DHHC2 is essential for ookinete morphogenesis and malaria transmission. Scientific Reports, 5, 16034.

Shen, B., Brown, K. M., Lee, T. D., \& Sibley, L. D. (2014). Efficient gene disruption in diverse strains of Toxoplasma gondii using CRISPR/CAS9. $\mathrm{mBio}, 5$, e01114.

Sidik, S. M., Huet, D., Ganesan, S. M., Huynh, M. H., Wang, T., Nasamu, A. S., ... Lourido, S. (2016). A genome-wide CRISPR screen in Toxoplasma identifies essential apicomplexan genes. Cell, 166, 1423-1435.e12.

Soldati, D., \& Boothroyd, J. C. (1993). Transient transfection and expression in the obligate intracellular parasite Toxoplasma gondii. Science, 260, 349-352.

Striepen, B., Crawford, M. J., Shaw, M. K., Tilney, L. G., Seeber, F., \& Roos, D. S. (2000). The plastid of Toxoplasma gondii is divided by association with the centrosomes. The Journal of Cell Biology, 151, 1423-1434.

Wetzel, J., Herrmann, S., Swapna, L. S., Prusty, D., John Peter, A. T., Kono, M., ... Gilberger, T. W. (2015). The role of palmitoylation for protein recruitment to the inner membrane complex of the malaria parasite. The Journal of Biological Chemistry, 290, 1712-1728.

Zhang, M., Wang, C., Otto, T. D., Oberstaller, J., Liao, X., Adapa, S. R., ... Adams, J. H. (2018). Uncovering the essential genes of the human malaria parasite Plasmodium falciparum by saturation mutagenesis. Science, 360, eaap7847.

Zhang, M. M., Wu, P.-Y. J., Kelly, F. D., Nurse, P., \& Hang, H. C. (2013). Quantitative control of protein S-palmitoylation regulates meiotic entry in fission yeast. PLoS Biology, 11, e1001597.

\section{SUPPORTING INFORMATION}

Additional supporting information may be found online in the Supporting Information section at the end of this article.

How to cite this article: Dogga SK, Frénal K. Two palmitoyl acyltransferases involved sequentially in the biogenesis of the inner membrane complex of Toxoplasma gondii. Cellular Microbiology. 2020;22:e13212. https://doi.org/10.1111/cmi. 13212 